\title{
HOXA repression is mediated by nucleoporin Nup93 assisted by its interactors Nup188 and Nup205
}

\author{
Ajay S. Labade, Krishanpal Karmodiya and Kundan Sengupta* (1)
}

\begin{abstract}
Background: The nuclear pore complex (NPC) mediates nuclear transport of RNA and proteins into and out of the nucleus. Certain nucleoporins have additional functions in chromatin organization and transcription regulation. Nup93 is a scaffold nucleoporin at the nuclear pore complex which is associated with human chromosomes 5, 7 and 16 and with the promoters of the HOXA gene as revealed by ChIP-on-chip studies using tiling microarrays for these chromosomes. However, the functional consequences of the association of Nup93 with HOXA is unknown.

Results: Here, we examined the association of Nup93 with the HOXA gene cluster and its consequences on HOXA gene expression in diploid colorectal cancer cells (DLD1). Nup93 showed a specific enrichment $\sim 1 \mathrm{~Kb}$ upstream of the transcription start site of each of the HOXA1, HOXA3 and HOXA5 promoters, respectively. Furthermore, the association of Nup93 with HOXA was assisted by its interacting partners Nup188 and Nup205. The depletion of the Nup93 sub-complex significantly upregulated HOXA gene expression levels. However, expression levels of a control gene locus (GLCCI1) on human chromosome 7 were unaffected. Three-dimensional fluorescence in situ hybridization (3D-FISH) analyses revealed that the depletion of the Nup93 sub-complex (but not Nup98) disengages the HOXA gene locus from the nuclear periphery, suggesting a potential role for Nup93 in tethering and repressing the HOXA gene cluster. Consistently, Nup93 knockdown increased active histone marks (H3K9ac), decreased repressive histone marks (H3K27me3) on the HOXA1 promoter and increased transcription elongation marks (H3K36me3) within the HOXA1 gene. Moreover, the combined depletion of Nup93 and CTCF (a known organizer of HOXA gene cluster) but not Nup93 alone, significantly increased GLCCI1 gene expression levels. Taken together, this suggests a novel role for Nup93 and its interactors in repressing the HOXA gene cluster.
\end{abstract}

Conclusions: This study reveals that the nucleoporin Nup93 assisted by its interactors Nup188 and Nup205 mediates the repression of HOXA gene expression.

Keywords: HOXA, Nuclear pore complex (NPC), Nup93, Nup188, Nup205, Nucleoporins (Nups), Chromatin, ChIP

\section{Background}

The nuclear pore complex (NPC) is a highly conserved protein complex, localized at the nuclear periphery and is required for import and export of proteins and RNA [1, 2]. Nucleoporins in Saccharomyces cerevisiae, Drosophila melanogaster and mammalian cells are also involved in transcriptional regulation [3-7], transcriptional memory

*Correspondence: kunsen@iiserpune.ac.in

Biology, Indian Institute of Science Education and Research (IISER),

Dr. Homi Bhabha Road, Pashan, Pune, Maharashtra 411008, India
[8-10], demarcating chromatin boundaries [11, 12], differentiation, development $[4,13-15]$, DNA damage repair [16, 17] and chromatin organization [18, 19]. These functions are likely to involve chromatin contacts with nucleoporins. Typically, Nups contact chromatin in either an off-pore or on-pore manner. In humans, Nup98 contacts chromatin in an off-pore manner inside the nucleoplasm away from the nuclear periphery [13, 20]. In Drosophila, mobile Nups such as Nup98, Sec13 and Nup50 re-localize to the nucleoplasm and contact chromatin [21]. Nup153 and Megator (Mtor) are mobile 
nucleoporins that associate with $\sim 25 \%$ of the Drosophila genome at Nucleoporin-associated regions (NARs) [22]. In neural progenitor cells, nucleoporins contact chromatin in an on-pore manner, for instance a group of genes that include GRIK1, NRG1 and MAP2 are specifically associated with Nup98 at the nuclear envelope upon transcriptional activation [13]. The yeast Nup170p associates with the RSC chromatin remodeling complex and the silencing factor Sir4p which cooperatively mediates the association of telomeres with the nuclear envelope resulting in sub-telomeric gene silencing [23]. Taken together, these studies suggest an association of nucleoporins with chromatin. However, the molecular mechanisms of nucleoporins and their interaction with chromatin in transcription regulation remain unclear. Nucleoporins in addition to their primary role in nuclear transport also function in chromatin organization. However, the mechanisms of chromatin organization mediated by stable and on-pore nucleoporins remain unclear.

The nucleoporin Nup93 sub-complex is composed of Nup93, Nup188, Nup205, Nup155 and Nup53 [24-28]. Nup93 is a highly stable nucleoporin with a relatively low dissociation rate from the nuclear pore complex $\left(K_{\text {off }}\right.$ : $4.0 \pm 3.4 \times 10^{-6} \mathrm{~s}^{-1}$ ) [29]. Interestingly, ChIP-chip studies using tilling microarrays for human chromosomes 5,7 and 16 in HeLa cells reveal that Nup93 contacts chromatin sub-domains on these chromosomes [30]. These studies show an association of Nup93 with the promoters of HOXA1, HOXA3 and HOXA5 on human chromosome 7. However, the potential role of nucleoporins in regulating HOX gene expression is unclear. This raises the intriguing possibility of Nup93 to function as an additional modulator of the HOXA chromatin sub-cluster and therefore HOXA gene expression during differentiation.

The HOXA gene locus (Chr.7p15.3) spans 150 kb of the sub-genomic region $(27,112,593-27,254,038 \mathrm{bp}$, hg19 assembly) and encodes for 11 transcription factors that are involved in pattern formation in early development [31]. Aberrant HOXA expression levels correlate with cancers and is dysregulated in breast carcinoma, human cutaneous melanoma and oral cancers [32-37]. Chromosome conformation capture studies have shown that the repressed HOXA gene cluster adopts a compact chromatin state organized as "multiple chromatin loops" for instance in undifferentiated NT2/D1 cells [38-40]. These loops of the HOXA gene loci are disrupted by the combined action of retinoic acid treatment and depletion of CTCF or PRC2 that transcriptionally activate HOXA gene expression [38].

Brown et al. [7] showed for the first time that Nup93 contacts chromatin and associates with the HOXA promoter. We extended this study to address the consequences of depleting Nup93 on HOXA gene expression. We show that Nup93 associates with and represses HOXA gene expression in a manner dependent on its interacting partners-Nup188 and Nup205, in diploid colorectal cancer cells (DLD1). The depletion of Nup93 or its interacting partners-Nup188 and Nup205, derepresses the HOXA gene cluster since this showed a marked increase in HOXA gene expression, facilitated by enhanced levels of the active histone marks (H3K9ac) and decreased levels of repressive histone marks (H3K27me3) on the HOXA1 promoter. In addition, transcription elongation marks (H3K36me3) were enriched within the HOXA1 gene. This is consistent with an untethering of the HOXA gene cluster from the nuclear periphery upon depletion of Nup93 or its interactors Nup188 or Nup205, but not in Nup98-depleted cells. Furthermore, Nup93 represses HOXA gene cluster independent of its key regulator, i.e., CTCF [38]. Taken together, this study reveals that Nup93 along with its interacting partners-Nup188 and Nup205, represses HOXA gene expression.

\section{Results}

It is well established that chromatin is associated with nuclear landmarks such as the nuclear lamina, nucleolus, nuclear bodies and the nuclear pore complex [41, 42]. However, the specific structural and functional role of these nuclear landmarks in regulating chromatin organization and gene expression remains elusive [42, 43]. As shown previously across biological systems, chromatin at the nuclear periphery is directly or indirectly associated with nuclear pore proteins [44]. Nup93-a scaffold nucleoporin at the core of the nuclear pore complex, contacts chromatin sub-domains of human chromosomes 5 , 7 and 16 in HeLa cells as revealed by ChIP-chip studies using tilling microarrays for these chromosomes (Fig. 1a) [30]. Furthermore, chromosome 7 is predominantly localized toward the nuclear periphery [45]. HOXA is an important gene cluster that maps to human chromosome 7 with Nup93 binding sites on HOXA1, HOXA3 and HOXA5 gene promoters [30]. The focus of our study was to examine the consequences of depleting Nup93 and its interactors on HOXA gene expression in diploid colorectal cancer cells (DLD1). The DLD1 cell line is a colorectal adenocarcinoma cell line which maintains a stable and near diploid karyotype with a modal number of 46 chromosomes.

\section{Nup93 associates with HOXA promoter regions}

Chromatin immunoprecipitation on chip in HeLa cells showed that Nup93 binds to the promoter regions [ 1000 bp upstream of the transcription start site (TSS)] of HOXA1, HOXA3 and HOXA5 on human Chr.7p15.2 
[30]. To validate the antibody that we used against Nup93, we performed immunoprecipitation assays, which detected a single band at $\sim 93 \mathrm{kDa}$ (Fig. 1b). Furthermore, siRNA-mediated knockdown followed by immunoblotting, showed a $>70 \%$ depletion of Nup93 (Fig. 1c), thereby validating this antibody for ChIP experiments. In order to ascertain if Nup93 associates with the HOXA promoter region and to extend earlier observations, we performed ChIP-qPCR of specific subregions of the HOXA promoter [30] (Fig. 1d). We found that Nup93 was indeed enriched on specific subregions of HOXA1, HOXA3 and HOXA5 promoters (Fig. 1d, e), which further validated that Nup93 associates with the HOXA1, HOXA3, and HOXA5 promoter sequences, respectively (Fig. 1e). Nup93 was not enriched on GLCCI1 promoter-a gene locus $19 \mathrm{Mb}$ upstream of HOXA1 on Chr.7p21.3, and therefore served as a negative control (Fig. 1e-iv) [30]. To further validate the association of Nup93 with HOXA promoter regions, we performed ChIP-qPCR with Nup93 in Nup93-depleted cells. ChIP-qPCR results showed a significantly reduced association of Nup93 with the HOXA1 promoter upon Nup93 knockdown (Fig. 1f). To ascertain the binding preferences of Nup93, we examined whether Nup93 associates with regions outside the HOXA1 promoter $(\sim 3 \mathrm{Kbp}$ upstream and downstream of the HOXA1 promoter region) (Fig. 1d) and another Nup93 target gene (GRM8) (Chr.7q31.11). Nup93 associates within the GRM8 gene body but not with its promoter [30]. We found that Nup93 does not associate with sites just outside the HOXA1 promoter $(\sim 3 \mathrm{Kbp})$ (Fig. 1g). In contrast, Nup93 was significantly enriched within the GRM8 gene (Fig. 1g). We reconfirmed our results by performing ChIP-PCR using primer pairs P3, P4, P2 and P4 for HOXA1, HOXA3, HOXA5 and GLCCI1 respectively (Fig. 1h). In summary, these results corroborate the association of Nup93 with the HOXA1, HOXA3 and HOXA5 promoter regions.

\section{Nup93 requires its interactors Nup188 and Nup205 to associate with the HOXA1 promoter}

We sought to determine whether the interactors of Nup93, i.e., Nup188 and Nup205 [46] are required for Nup93 to associate with the HOXA1 promoter region. We ascertained if Nup93 interacts with Nup188 and Nup205. Co-immunoprecipitation of Nup93 from wholecell extracts of DLD1 cells showed that Nup93 interacts with Nup188 and Nup205, respectively (Fig. 2a, b) [46, 47]. However, Nup93 does not associate with Nup98 and neither did we detect an association between Nup188 and Nup98 (Fig. 2a, b), consistent with previous findings that Nup98 does not interact with Nup93 [46]. Reverse co-immunoprecipitation showed that Nup188 associates with Nup93 but not with Nup205 or Nup98 (Fig. 2b). Furthermore, Co-IP studies showed a reduced interaction of Nup93 with Nup188 in Nup205-depleted cells, suggesting the requirement for Nup205 in the interaction between Nup93 and Nup188 (Additional file 1: Fig. S1a). Taken together, these results suggest that Nup93 associates with Nup188 and Nup205 consistent with co-immunoprecipitation assays performed in $C$. elegans and $S$. cerevisiae, which show that Nup93 interacts with nucleoporins-Nup188 and Nup205 [26, 27, 46-48].

We determined whether the interactors of Nup93, i.e., Nup188 and Nup205 are required for Nup93 to associate with the HOXA1 promoter region (Fig. 2d). We performed ChIP with Nup93, in a background of Nup188- or Nup205-depleted DLD1 cells (Fig. 2c). ChIP-PCR and chip-qPCR performed on HOXA1 promoter region (Region P3, Fig. 1d, e-i) showed that Nup188 and Nup205 depletion decreased the occupancy of Nup93 by $70 \%$ on the HOXA1 promoter (Fig. $2 \mathrm{~d}$ and Additional file 1: Fig. S1b). However, the occupancy of the core histone H3 (ChIP with anti-PanH3 antibody that detects core histone $\mathrm{H} 3$ ) was unaltered on either HOXA1 or GLCCI1 promoters upon Nup188 or Nup205

\footnotetext{
(See figure on next page.)
}

Fig. 1 Nup93 associates with HOXA1, HOXA3 and HOXA5 promoters. a Pictorial representation of the nuclear pore complex (NPC) showing the relative location of the Nup93 sub-complex, and Nup98 within the nuclear pore complex [103]. b Immunoprecipitation of Nup93 using anti-Nup93 antibody on whole-cell extracts of DLD1 cells (a representative full blot from three independent biological replicates, $N=3$ ). Anti-rabbit heavy chain IgG shows equal precipitation efficiency in IgG and Nup93 lanes. c A representative full Western blot showing siRNA-mediated depletion of Nup93 in DLD1 cells (lane Nup93 Kd), Untreated Untreated cells, siNeg ON-TARGETplus non-targeting siRNA control (a representative blot from three independent biological replicates, $N=3$ ). d Pictorial representation of primer pair positions (P1-P4) on the promoter of HOXA1, HOXA3 and HOXA5 genes, respectively, upstream region and downstream region indicates primer position outside the HOXA1 promoter, arrow indicates transcription start site (TSS). e ChIP experiments were performed using antibodies specific to Nup93 and lgG control. Nup93 ChIP-qPCR on (i) HOXA1 (ii) HOXA3 (iii) HOXA5 and (iv) GLCCI1 promoters, respectively. Y-axis: immunoprecipitated DNA relative to 1\% input, corrected for ChIP using non-specific $\lg G(N=2$, data from two independent biological replicates that include a total of six technical replicates), error bar standard error of mean (SEM). f Nup93 ChIP-qPCR was performed in untreated and Nup93 knockdown cells for HOXA1 promoter using primer pairs P1-P4. g Nup93 ChIP-qPCR using primer pairs outside HOXA1 promoter regions (upstream region and downstream region) and primers for a Nup93-associated gene (GRM8), used as a positive control. h ChIP-PCR amplification of HOXA1, HOXA3 and HOXA5. GLCCI1 used as negative control. Nup93 binds to 300-600 bp on each of these HOXA promoters, ( $N=2$, representative data from two independent biological replicates) 


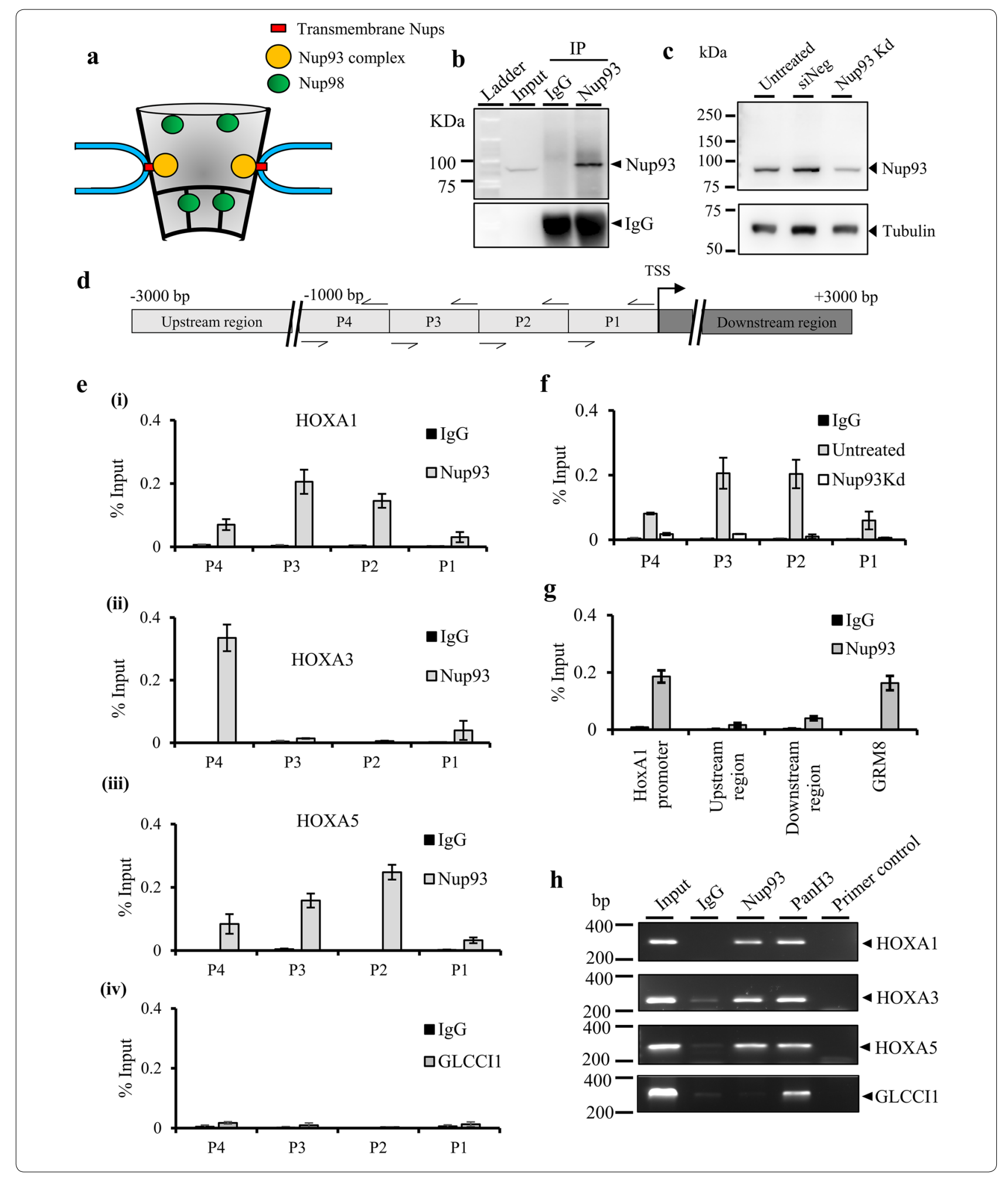




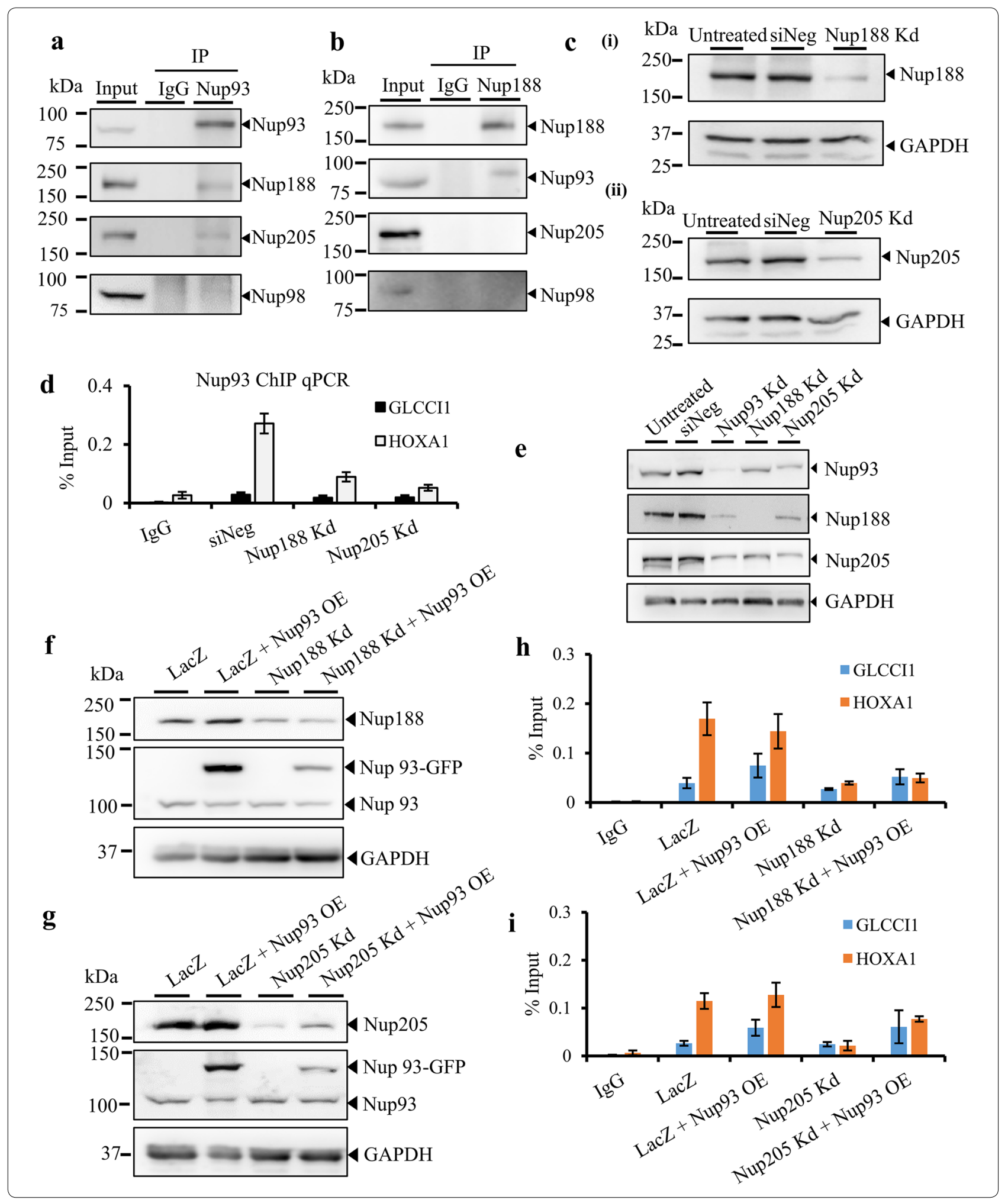


(See figure on previous page.)

Fig. 2 Nup93 interacts with Nup188 and Nup205 and associates with the HOXA1 promoter. a, b Immunoprecipitation was performed using antibodies specific for (a) Nup93; (b) Nup188 and lgG followed by Western blotting for Nup93, Nup188, Nup205 and negative control—Nup98 (representative data from three independent biological replicates, $N=3$, single experiment for Nup98). c (i) Nup188 and (ii) Nup205 were knocked down in DLD1 cells using siRNA. A representative Western blot showing the extent of knockdown (representative Western blot from three independent biological replicates, $N=3$ ). d ChIP experiment was performed using an anti-Nup93 antibody in untreated, non-targeting siRNA control (siNeg), Nup188 Kd (Knockdown) and Nup205 Kd cells. ChIP-qPCR analysis was used to determine the extent of Nup93 association with the HOXA1 promoter in Nup188 and Nup205 knockdown cells (Input and PanH3 in Fig. 2 d are from Nup205 Kd sample) Y-axis: immunoprecipitated DNA relative to $1 \%$ input, corrected for ChIP using non-specific lgG ( $N=2$, data from two independent biological replicates that include a total of six technical replicates), error bar: standard error of mean (SEM). e A representative Western blot showing the effect of Nup93, Nup188 and Nup205 depletion on one another (three independent biological replicates, $N=3$ ), $\mathbf{f}, \mathbf{g}$ a representative Western blot showing overexpression of Nup93 upon Nup188 (f) and Nup205 knockdown (g). GAPDH was used as a loading control. h, i ChIP-qPCR was performed upon overexpression of (f) Nup93 in Nup188and (g) Nup205-depleted cells. Y-axis: immunoprecipitated DNA relative to 1\% input, corrected for ChIP using non-specific lgG ( $N=2$, data from two independent biological replicates that include a total of six technical replicates), error bar: standard error of mean (SEM)

depletion (Additional file 1: Fig. S1b-c). Although independent knockdowns of Nup93, Nup188 and Nup205 did not affect the transcript levels of one another (Additional file 1: Fig. S1d), we detected a decrease in their relative protein levels in an interdependent manner (Fig. 2e and Additional file 1: Fig. S1f-g). We therefore considered the possibility that the reduced occupancy of Nup93 on HOXA1 could be attributed to reduced levels of Nup93 in cells depleted of Nup188 or Nup205. To account for the decrease in the levels of Nup93 upon Nup188 or Nup205 depletion, we overexpressed Nup93 in a background of Nup188 or Nup205 depletion (Fig. 2f, g). Despite Nup93 overexpression (Fig. 2f, g, Additional file 1: Fig. S1e), Nup93 showed a reduced occupancy on the HOXA1 promoter in either Nup188- or Nup205depleted cells (Fig. 2h, i). This suggests that overexpressed Nup93 is unable to associate with the HOXA1 promoter in the absence of Nup188 or Nup205. Taken together, these results suggest that a stable complex of Nup188-Nup93-Nup205 is required for Nup93 to associate with the HOXA1 promoter.

\section{HOXA gene expression is upregulated in Nup93-, Nup188- or Nup205-depleted cells}

The finding that Nup93 associates with the promoters of HOXA1, HOXA3 and HOXA5 genes assisted by Nup188 and Nup205 (Figs. 1e, 2d) prompted us to investigate whether Nup93 and its interactors regulate HOXA gene expression. We independently knocked down Nup93, Nup188 and Nup205 in DLD1 cells and assessed expression levels of all genes within the HOXA gene cluster (HOXA1-HOXA13) by qRT-PCR (Fig. 3ac). Nup93, Nup188 and Nup205 knockdown showed a $>80 \%$ reduction in their transcript levels (Fig. 3a-c, arrow). Remarkably, the transcript levels of HOXA genes (HOXA1, HOXA3, HOXA5 and HOXA9) were strikingly upregulated (fold change $>$ twofold) upon Nup93 Kd (Fig. 3a). HOXA1 showed an increase in transcript levels in all three nucleoporin knockdowns to four-sixfold, suggesting a significantly greater impact on HOXA1 expression levels upon Nup93, Nup188 and Nup205 knockdowns. Furthermore, HOXA1, HOXA3, HOXA5 and HOXA9 were significantly upregulated in Nup188and Nup205-depleted cells (Fig. 3b, c). Interestingly, the expression levels of HOXA13 and GLCCI1 were unaffected in all the three Nup knockdowns (HOXA13, GLCCI1, Fig. 3a-c). We also used two independent siRNA oligonucleotides to knockdown Nup93 (Additional file 2: Fig. S2a), which showed an upregulation of HOXA1, HOXA3, HOXA5 and HOXA9 genes but not of HOXA13 or GLCCI1 (Fig. S2b), consistent with previous results (Fig. 3a). Of note, the depletion of Nup98 did not alter gene expression levels of the HOXA gene cluster and GLCC1 (Additional file 2: Fig. S2b), further suggesting a novel role for Nup93 and its interacting partners-Nup188 and Nup205, in regulating HOXA gene expression in DLD1 cells.

Since ChIP showed a reduced occupancy of Nup93 on the HOXA1 promoter despite Nup93 overexpression in Nup188- and Nup205-depleted cells (Fig. 2h, i), we determined the effect of Nup93 overexpression on HOXA transcript levels in Nup188- and Nup205-depleted cells, respectively. Interestingly, Nup93 overexpression was incapable of rescuing HOXA gene expression in Nup188and Nup205-depleted cells (Fig. 3d, e, Additional file 1: Fig. S1e). Taken together, these assays further reiterate a requirement for Nup188 and Nup205 in Nup93-mediated repression of HOXA gene expression.

We were curious to examine the consequences of allowing cells to recover after Nup93 depletion and asked whether the upregulation of HOXA gene expression was reversible or irreversible, since an irreversible upregulation of HOXA may imply a feedback effect to sustain HOXA upregulation. We allowed cells to recover in culture for 8 days after 48 h of Nup93, Nup188 and Nup205 knockdown in DLD1 cells. Interestingly, we detected a significant restoration in the transcript levels of Nup93, Nup188 and Nup205, respectively (Additional 


\section{$a$ हु}

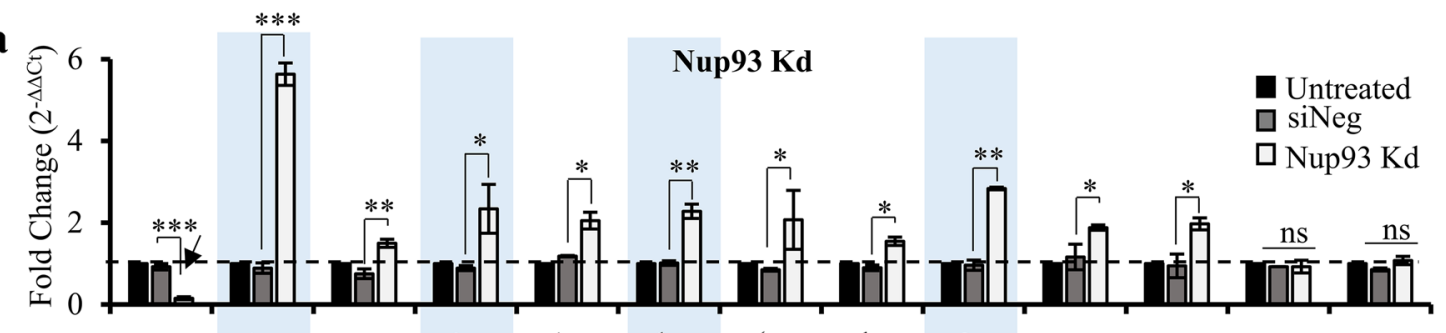

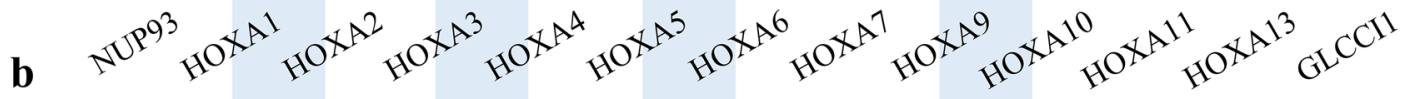
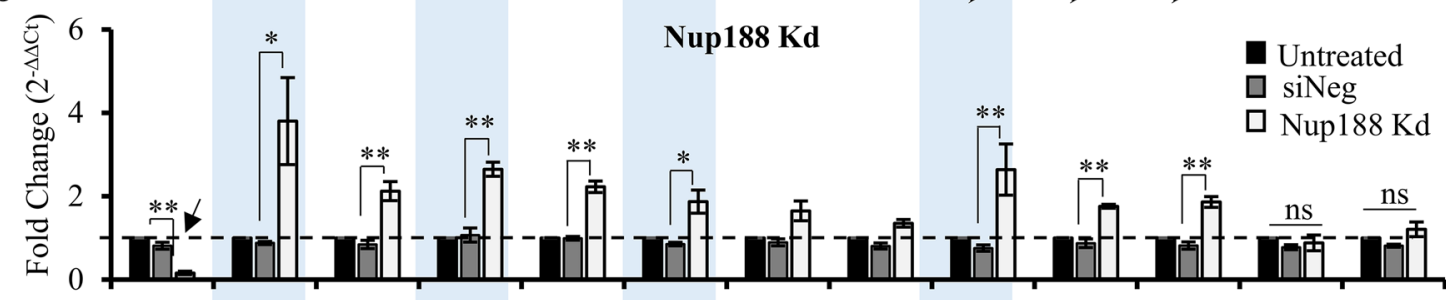

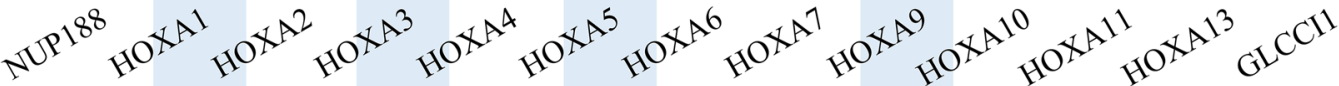

c

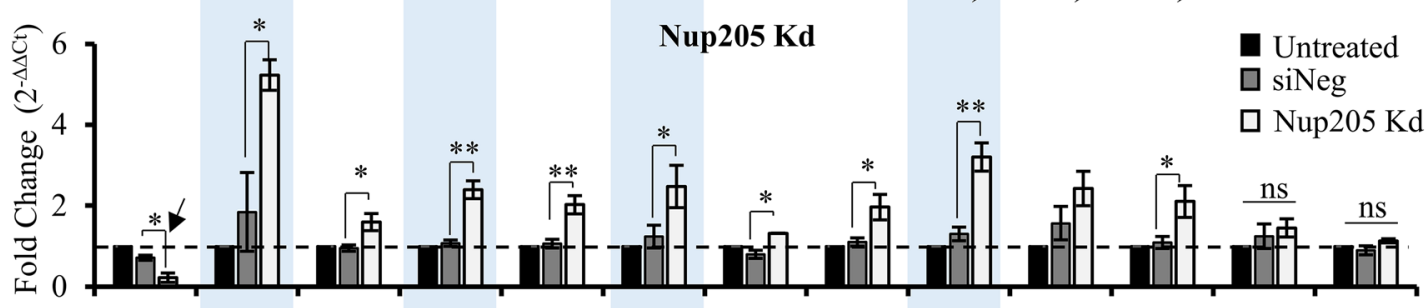

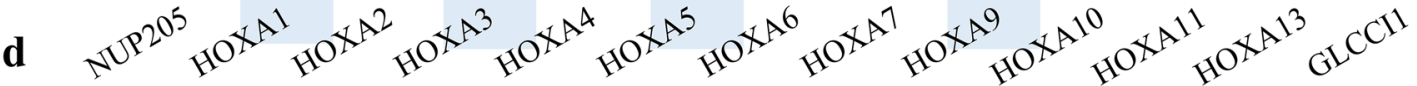

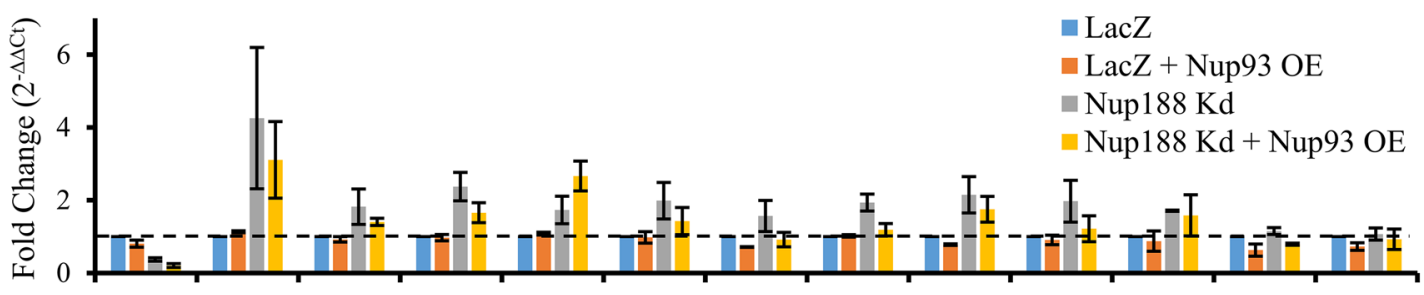

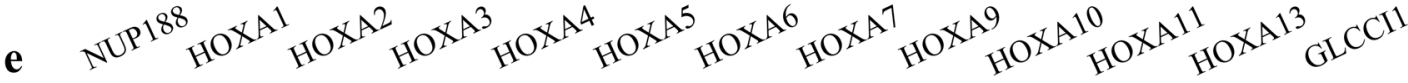

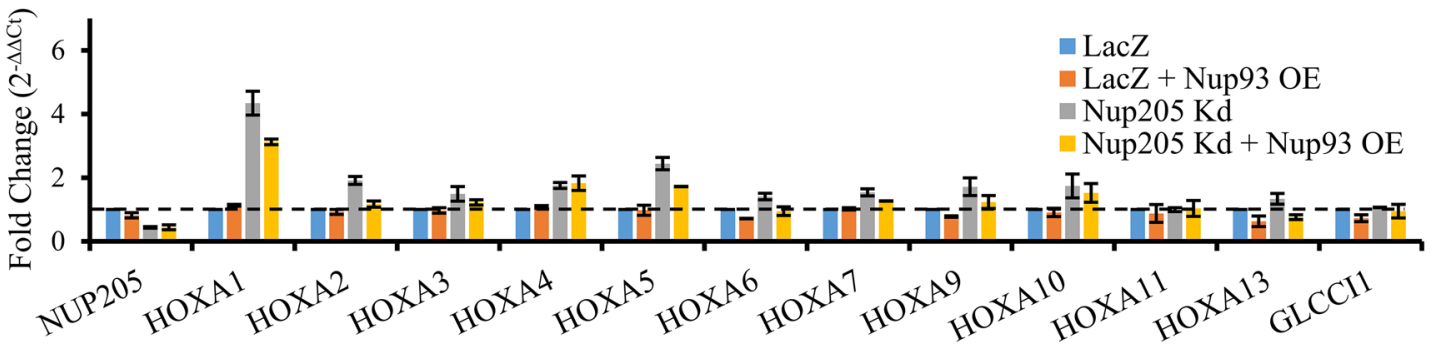

Fig. 3 Depletion of Nup93, Nup188 and Nup205 derepresses HOXA gene cluster. a-c qRT-PCR analyses was used to determine mRNA levels of all HOXA genes (HOXA1 to HOXA13) upon (a) Nup93, (b) Nup188 and (c) Nup205 knockdowns in DLD1 cells. Graph represents fold change (2- ${ }^{\left.-\Delta \Delta C_{\mathrm{t}}\right)}$ in levels of mRNA normalized to untreated cells. Error bars: SEM, data from three independent biological replicates that include total of nine technical replicates, ${ }^{*} p<0.05 ;{ }^{* *} p<0.01 ;{ }^{* * *} p<0.001$ (Students $t$ test between siNeg and knockdown). GLCCl, served as a negative control. d ,e qRT-PCR analyses was used to determine mRNA levels of all HOXA genes (HOXA1 to HOXA13) upon Nup93 overexpression in (d) Nup188- and (e) Nup205depleted cells. Graph represents fold change $\left(2^{-\Delta \Delta c_{t}}\right)$ in levels of mRNA normalized to untreated cells. Error bars: SEM, data from two independent biological replicates that include total of six technical replicates. GLCCI, served as a negative control 
file 2: Fig. S2c-e) accompanied by the downregulation of HOXA1 and HOX9 transcript levels, while HOXA13 levels remained unaltered (Additional file 2: Fig. S2c-e). Taken together, these results uncover a novel role for Nup93 and its interacting partners in the repression of the HOXA gene cluster.

\section{HOXA gene loci is untethered from the nuclear periphery in Nup93-, Nup188- and Nup205-depleted cells}

The significant upregulation of HOXA genes upon Nup93, Nup188 and Nup205 depletion prompted us to determine the spatial localization of HOXA gene locus upon Nup93, Nup188 or Nup205 depletion in the interphase nucleus. Gene loci tethered to the nuclear periphery are typically maintained in a state of repression [49]. We performed three-dimensional fluorescence in situ hybridization (3D-FISH) followed by confocal imaging of fluorescently labeled HOXA gene locus and Chromosome 7 Territories (CT7) in cells independently depleted of Nup93, Nup188 and Nup205, respectively (Fig. 4a). We measured the shortest distance of the HOXA gene locus from the nuclear periphery [50]. HOXA gene loci were predominantly localized closer to the nuclear periphery in control cells $[$ median $=0.64 \mu \mathrm{m}$ from the edge of the nucleus in control cells (Fig. 4b, c)]. Interestingly, Nup93-, Nup188- or Nup205-depleted cells showed an $\sim 0.2-$ $\mu \mathrm{m}$ shift of the HOXA gene loci away from the nuclear edge (Fig. 4b, c). In contrast, Nup98 depletion did not affect the positioning of the HOXA loci with respect to the nuclear periphery (Fig. 4b, c). Taken together, this suggests that Nup93 and its interactors are likely to tether the HOXA gene locus closer to the nuclear periphery in order to maintain HOXA gene locus in a repressed configuration, while depletion of Nup93 or either of its interacting partners results in a movement of the HOXA gene locus toward the nuclear interior (Fig. 4c).

\section{Nup93 depletion alters the relative occupancy of histone marks on the HOXA1 promoter}

We determined whether the observed derepression of the HOXA gene cluster upon Nup93 depletion is associated with an altered occupancy of active and inactive histone marks on the HOXA1 promoter region (Fig. 5a). We performed ChIP with active (H3K9ac) and repressive (H3K27me3) histone marks on the HOXA1 promoter in Nup93-depleted cells (Fig. 5b-e). We determined the levels of active and repressive histone marks on the HOXA1 promoter upon Nup93 knockdown, since HOXA1 showed the highest increase in transcript levels (>four-sixfold) in cells depleted of Nup93 or its interacting partners-Nup188 or Nup205 (Fig. 3a-c). We performed ChIP with antibodies against active (H3K9ac) and repressive (H3K27me3) histone marks followed by ChIP-qPCR with overlapping primers $\sim 1 \mathrm{~Kb}$ upstream of the HOXA1 transcription start site (Fig. 5a). We detected a significant enrichment of the active histone mark (H3K9ac), 1 Kb upstream of the transcription start site of the HOXA1 gene (Fig. 5b) and a marked decrease in the occupancy of the repressive histone mark (H3K27me3) upon Nup93 knockdown (Fig. 5c). Notably, the relative levels of active and repressive histone marks were unaltered upon Nup93 depletion on the promoters of control genes-GAPDH and GLCCI1 (Fig. 5d, e). Furthermore, the total levels of H3K9ac, H3K27me3 and PanH3 were unaffected upon Nup93 depletion in DLD1 cells (Fig. 5f). Taken together, these results suggest a strong correlation between Nup93 depletion and the relative enrichment of the active histone mark and decreased occupancy of the inactive histone mark on the HOXA1 promoter. This is consistent with previous studies which show that silencing of the HOXA gene cluster is mediated by Polycomb (PcG) proteins by the recruitment of repressive histone marks (H3K27me3) on the HOXA gene promoter of NT2/D1 embryonal carcinoma cell line and in HeLa cells $[38,51]$.

Since we detected a striking increase in the expression levels of the HOXA1 gene upon Nup93, Nup188 and Nup205 knockdown, we asked whether HOXA1 gene expression correlates with active transcriptional elongation, i.e., active transcription of the HOXA1 gene. We performed ChIP with H3K36me3-a histone mark enriched during transcriptional elongation [52], with the HOXA1 gene (Fig. 5a). Interestingly, Nup93 knockdown

\footnotetext{
(See figure on next page.)

Fig. 4 HOXA gene loci is untethered from the nuclear periphery upon Nup93, Nup188 and Nup205 depletion. a Representative images (maximum intensity projection of a confocal image stack) of 3D-FISH for HOXA (red), CT7 (green) and DAPI (blue) performed on siLacZ-, Nup93-, Nup188-, Nup205- and Nup98-depleted DLD1 cells. Scale bar 10 $\mu \mathrm{m}$, white dotted line indicates nuclear boundary. b Dot scatter plot showing shortest distance of HOXA gene locus from nuclear periphery demarcated by DAPI in siLacZ ( $n=164$ loci signals)-, Nup93 $(n=154)-$, Nup 188 ( $n=178)-$, Nup205 ( $n=178$ )- and Nup98 ( $n=124)$-depleted DLD1 cells, horizontal bar represents median with interquartile range. Data from two independent biological replicates, ${ }^{* *} p<0.01 ;{ }^{* * *} p<0.001$ (Kolmogorov-Smirnov test). c \% Frequency distribution profile of HOXA gene locus from nuclear periphery plotted as bins of $\sim 0.2 \mu \mathrm{m}$ each from the nuclear periphery. $Y$-axis represents $\%$ frequency of HOXA locus pooled from two independent biological replicates
} 
a
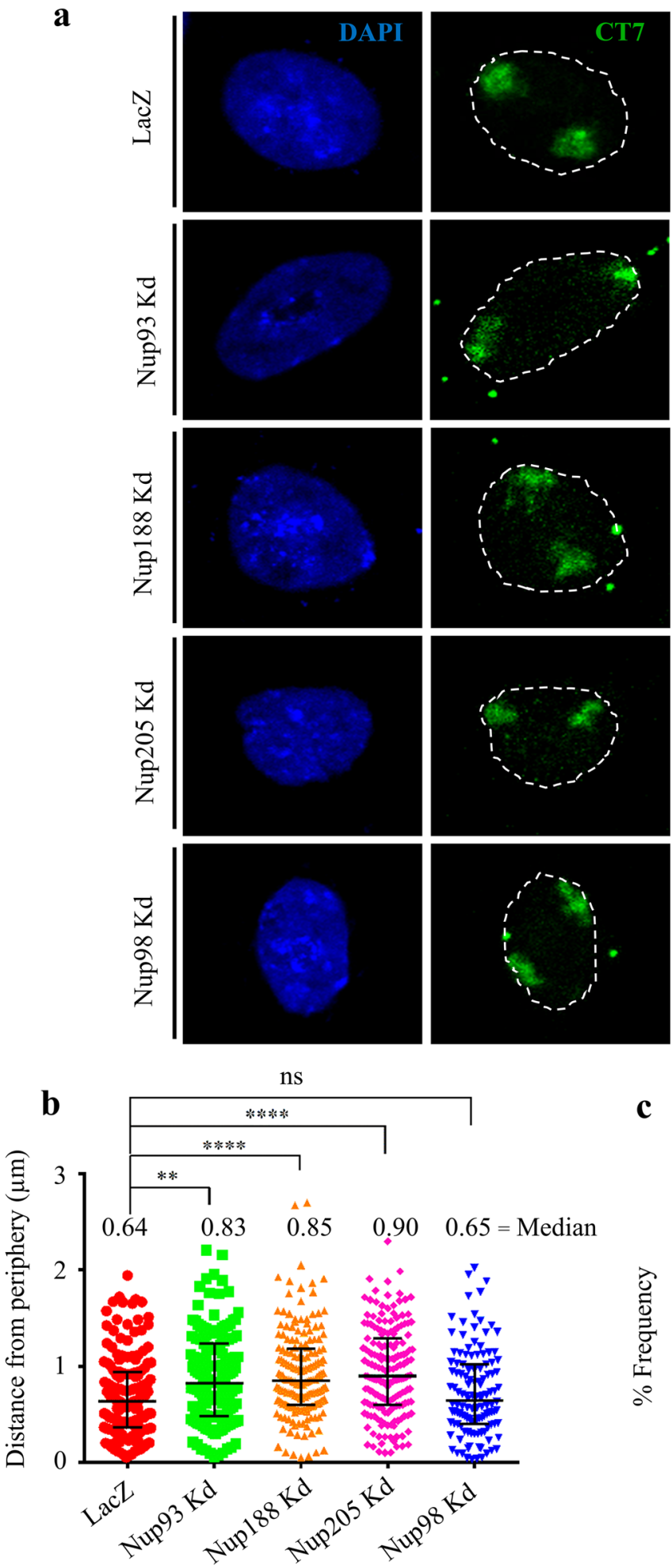

c
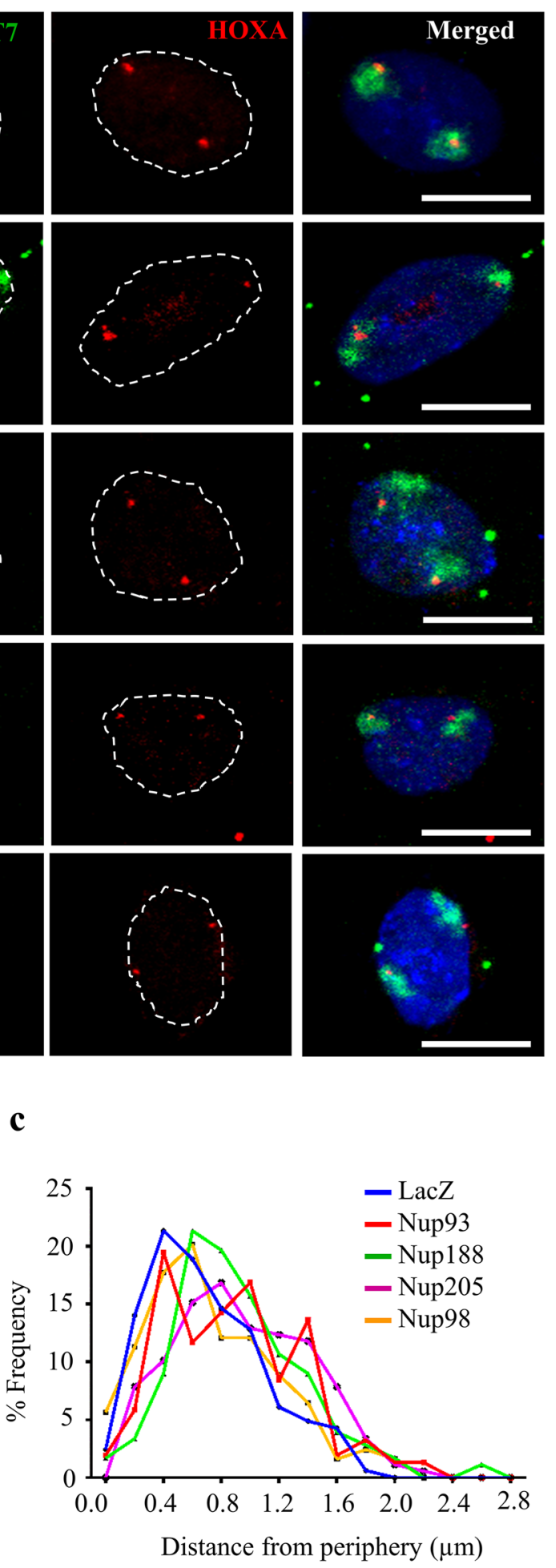

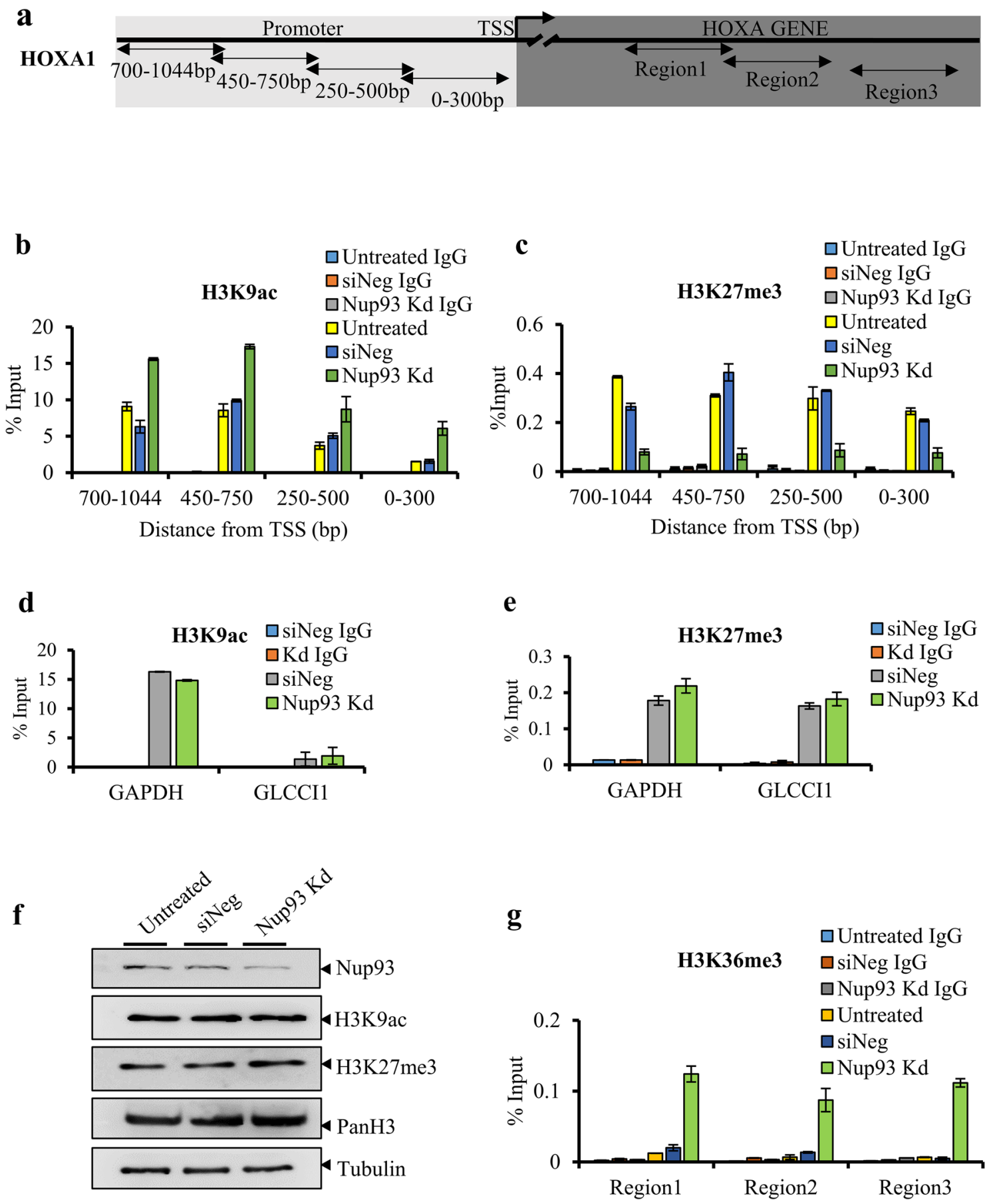

Fig. 5 Nup93 depletion alters the occupancy of histone marks on HOXA1 promoter. a Pictorial representation of the HOXA1 promoter and regions within the HOXA1 gene (Region 1-Region 3). Left (light gray) promoter of HOXA1 gene, double arrowheads: overlapping primer positions on HOXA1 promoter. Right (dark gray) regions within HOXA1 gene, double arrowheads: ChIP-qPCR primer positions within HOXA1 gene (Region 1-Region 3). b, c ChIP experiments were performed using antibodies specific to (b) H3K9ac, (c) H3K27me3 and lgG in untreated, siNeg and Nup93 knockdown cells (IgG is below detection limit $<0.2 \%$ of input in 'b-d', re-plotted in Additional file 2: Fig. S2h-j), d, e GAPDH promoter and GLCCI1 promoter were used as positive and negative controls, respectively. Y-axis: immunoprecipitated DNA is relative to $1 \%$ input, corrected for ChIP using non-specific IgG (data from two independent biological replicates that include a total of six technical replicates), error bars: SEM. f Representative Western blot of untreated, siNeg and Nup93 Kd cells showing that total levels of H3K9ac and H3K27me3 are unaltered. PanH3 and Tubulin were used as loading controls (data from a single experiment). g Elongation mark (H3K36me3) shows increased occupancy on the HOXA1 gene (Region 1, Region 2 and Region 3), data from two independent biological replicates that include a total of six technical replicates, error bars: SEM, Nup93 knockdown alters occupancy of histone marks on HOXA1 promoter 
showed a specific enrichment of H3K36me3 on all three regions (Region 1-Region 3) within the HOXA1 gene (Fig. 5g). Taken together, these experiments strongly suggest that active transcription of the HOXA1 gene upon depletion of Nup93 is associated with an increased occupancy of active histone marks and decreased levels of repressive histone marks.

\section{Nup93 depletion reduces nuclear import but does not affect nuclear export}

In order to address the role of Nup93 and its interactors in HOXA gene expression, we were curious to determine whether nuclear transport was affected in cells depleted of Nup93 or either of its interactors Nup188 or Nup205. To determine the effect of Nup93 depletion on nuclear import, we transfected DLD1 cells with a dexamethasone-inducible reporter construct consisting of the hormone-responsive domain of glucocorticoid and GFP fused to the M9 core domain (GR2-GFP2-M9core) [53]. Upon transient transfection, the reporter construct was exclusively localized in the cytoplasm, which translocated to the nucleus within $30 \mathrm{~min}$ in the presence of $5 \mu \mathrm{M}$ dexamethasone (glucocorticoid hormone analogue) in control cells (LacZ, Fig. 6a and c, Additional file 3: Fig. S3). Nup93, Nup188 or Nup205 depletion showed a reduced nuclear import of the reporter GFP construct as compared to control cells after $30 \mathrm{~min}$ of dexamethasone addition (Fig. 6a, c, Additional file 3: Fig. S3). Notably, Nup98 depletion blocked nuclear import of the reporter GFP (Fig. 6c and Additional file 3: Fig. S3). Taken together, this suggests that the depletion of Nup93 or its interactors results in reduced nuclear import.

As a readout of nuclear export, we examined the nucleocytoplasmic distribution of fluorescently labeled Poly(A) RNA in Nup93-, Nup188- or Nup205-depleted cells (Fig. 6b, d), since Poly(A) RNA is abundant in cells and is typically associated with mature transcripts [54]. Poly(A)
RNA is detectable within the nucleus as foci (Fig. 6b) and diffusely within the cytoplasm (Fig. 6b, siNeg panel). We did not detect a significantly altered distribution of Poly(A) signals inside or outside the nucleus in Nup93-, Nup188- or Nup205-depleted cells, as compared to control cells (siNeg, Fig. 6b, d, Additional file 4: Fig. S4). This is consistent with a normal nuclear export in nuclear reassembly assays performed in Nup188-Nup93 immunodepleted Xenopus egg extracts [46]. Depletion of Nup98-an established regulator of nuclear export, showed a retention of Poly(A) RNA in the nucleus, evidenced by a significant increase in the nuclear-to-cytoplasmic ratio of Poly(A) RNA in DLD1 cells (Fig. 6b, d) [55-57]. Taken together, these assays suggest that, although nuclear export was not significantly affected, nuclear import was reduced but not inhibited in cells depleted of Nup93 or its interacting partners.

\section{HOXA derepression upon Nup93 depletion is independent of CTCF}

CTCF and PRC2 complex proteins regulate chromatin looping and expression of the HOXA gene cluster in embryonic stem cells [31, 38, 39, 51, 58-60]. We sought to ask whether CTCF regulates HOXA gene expression levels. We determined whether CTCF and PRC2 complex proteins associate with Nup93 (Fig. 7a, b). Co-immunoprecipitation (Co-IP) assays did not reveal an association between Nup93 and CTCF (Fig. 7a), or between Nup93 and PRC2 complex proteins (EED or Suz12) in DLD1 cells (Fig. 7b). Furthermore, levels of CTCF or the PRC2 complex proteins (EZH2, Suz12 and EED) were unaltered in Nup93-, Nup188- or Nup205-depleted cells (Fig. 7c). CTCF is a known organizer of the HOXA gene cluster and has conserved binding sites that are proximal to the 5 ' region of the HOXA gene cluster which do not overlap with Nup93 binding sites (Fig. 7e) [38]. We therefore investigated the effect of CTCF depletion on HOXA gene

\footnotetext{
(See figure on next page.)

Fig. 6 Nup93 depletion reduces nuclear import but does not affect nuclear export. a A representative image of nuclear import assay performed using GR2-GFP2-M9 construct transfected in cells treated independently with LacZ and siNup93. To induce nuclear import of GR2-GFP2-M9 fusion protein, cells were treated with dexamethasone (Dex) (5 $\mu \mathrm{M}$ ) for $30 \mathrm{~min}$, white arrowhead indicates absence of cytoplasmic GFP in LacZ + Dex and residual cytoplasmic GFP in Nup93 Kd + Dex. Scale bar $\sim 10 \mu \mathrm{m}$. b A representative image of Poly(A) RNA FISH performed using FAM-labeled oligo(dT) probe (green) in siNeg, Nup93 Kd, Nup188 Kd, Nup205 Kd and Nup98 Kd, scale bar $10 \mu \mathrm{m}$, white arrowhead indicates Poly(A) RNA foci in the nucleus. Nuclear boundary is marked by dotted line in enlarged panel. Nup98 enlarged panel shows both nuclear and cell boundary with white dotted line. c Nuclear/cytoplasmic (N/C) ratio of GR2-GFP2-M9 was determined by quantifying its relative fluorescence intensity in the nucleus and cytoplasm. Scatter plot of GFP signals expressed as nuclear-to-cytoplasmic ratios from LacZ ( $n=60$ cells), Nup93 Kd ( $n=57)$, Nup188 Kd $(n=60)$, Nup205 Kd $(n=59)$ and Nup98 Kd $(n=60)$, data from 2 independent biological replicates $(* * * * 00.0001)$. d Poly(A) RNA distribution was determined by quantifying its fluorescence intensity in the nucleus and cytoplasm. Scatter plot of Poly(A) signals expressed as nuclear (N)-tocytoplasmic (C) ratios from siNeg ( $n=127$ cells), Nup93 Kd $(n=158)$, Nup188 Kd ( $n=288)$, Nup205 Kd ( $n=288$ ); N/C ratio was not significant (ns) when compared to siNeg $(p>0.05)$, while Nup98 Kd $(n=97)$ shows a relatively higher nuclear-to-cytoplasmic ratio (N/C ratio) of Poly(A) signals $\left({ }^{* * *} p=0.0017\right)$. Two independent biological replicates for siNeg, Nup93 Kd and Nup98 Kd. Data from a single experiment for Nup188 Kd and Nup205 Kd. Horizontal line represents median, $p$ values obtained from Mann-Whitney U test. Nuclear transport of Poly(A) RNA was unaffected in Nup93-, Nup188- or Nup205- depleted cells
} 
a

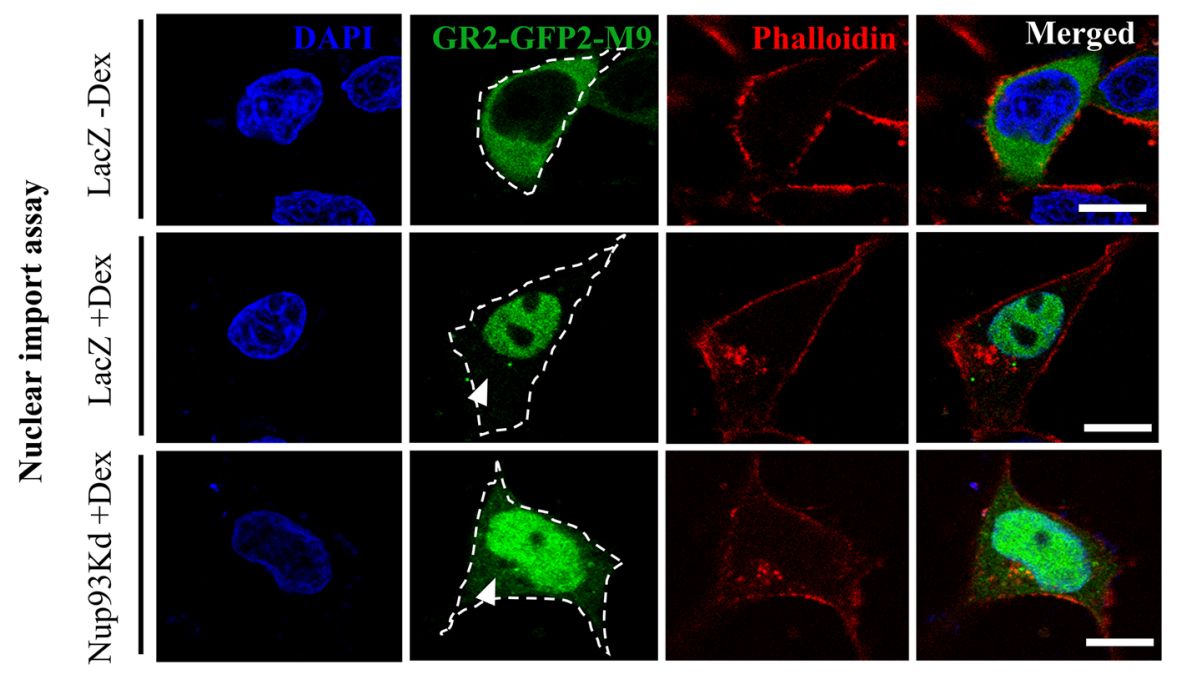

b

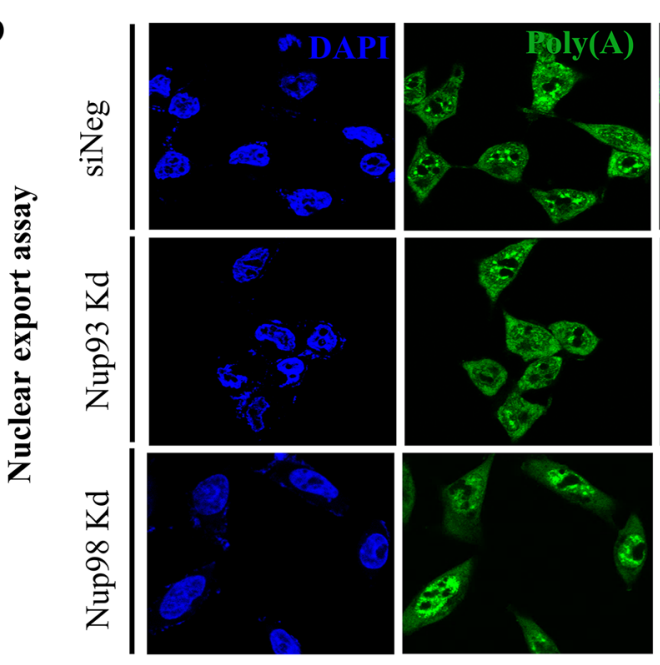

c

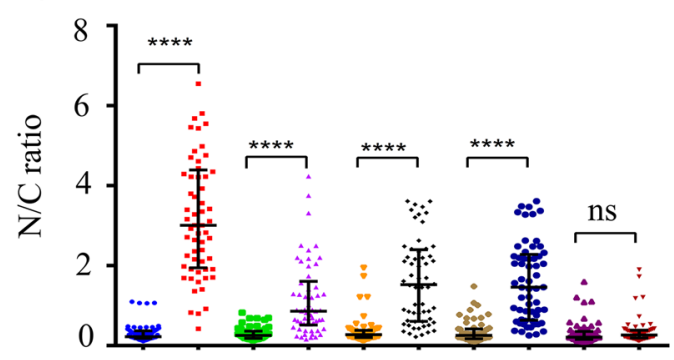

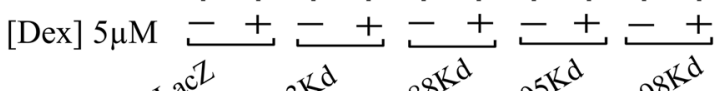

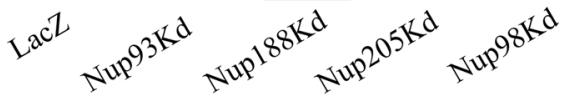
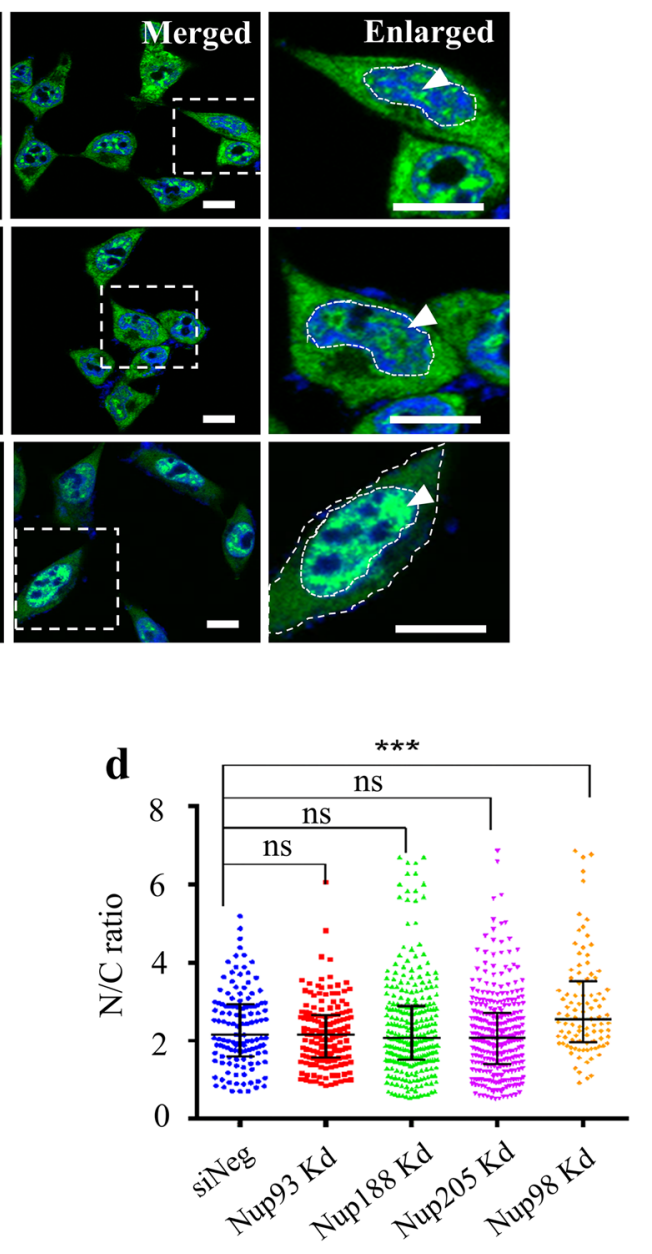

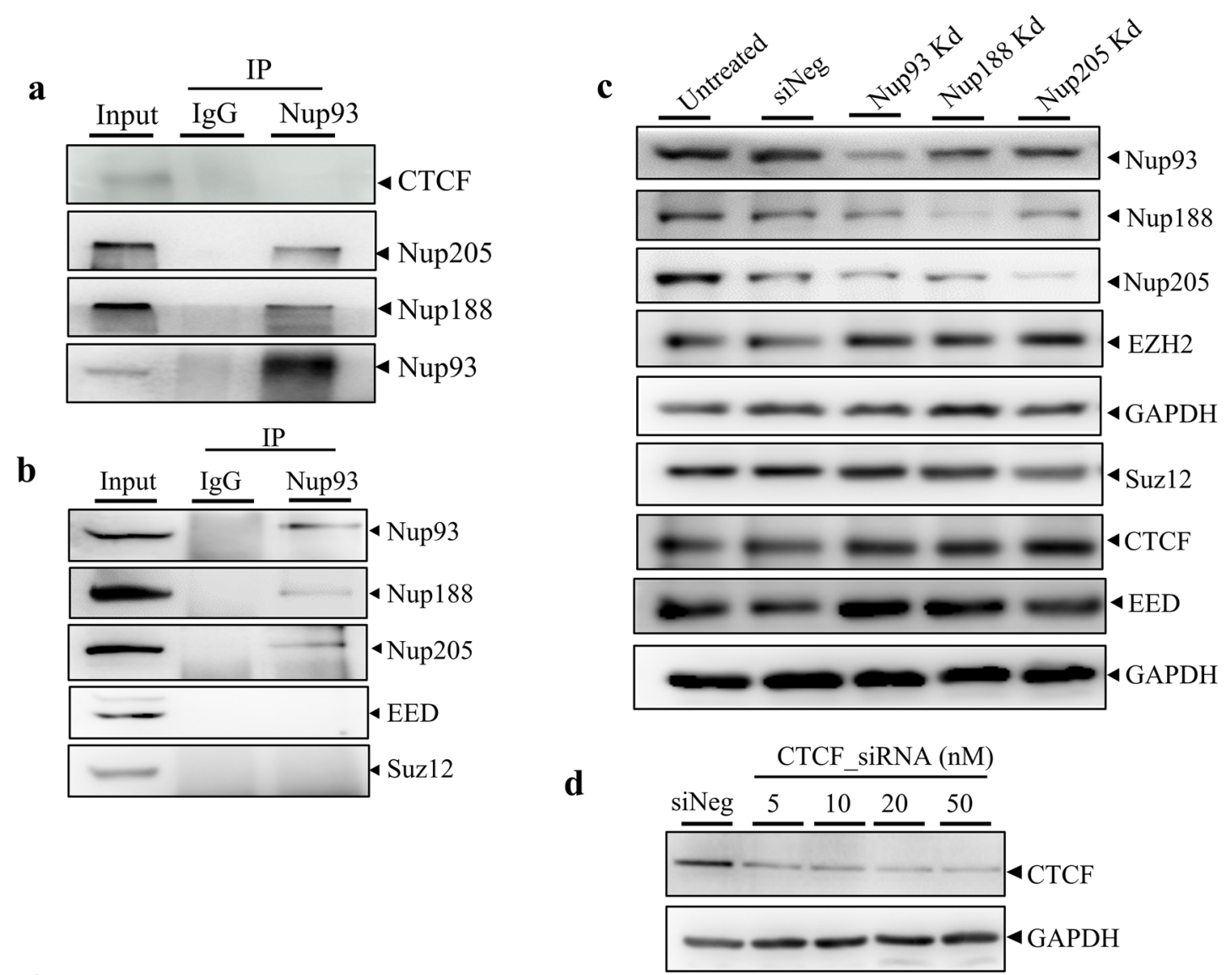

e
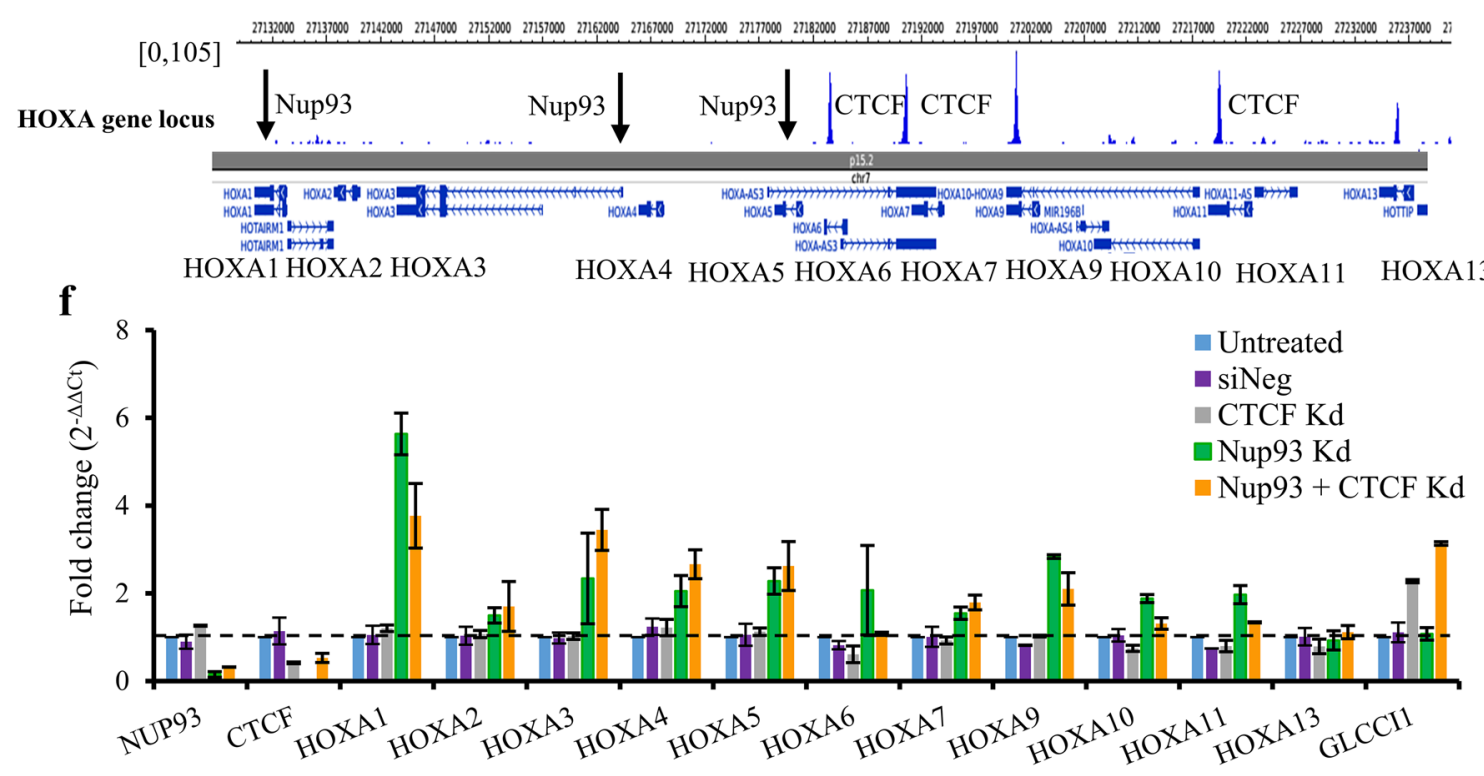


\begin{abstract}
(See figure on previous page.)
Fig. 7 HOXA is upregulated upon Nup93 depletion independent of CTCF. a Co-immunoprecipitation was performed using anti-Nup93 antibody and negative control lgG followed by Western blotting for CTCF, Nup205, Nup188 and Nup93 (data from two independent biological replicates, $N=2$ ), b Co-IP for Nup93 and Western blot for Nup93, Nup188, Nup205 (data from three independent biological replicates, $N=3$ ), PRC2 complex proteins EED and Suz12 (data from a single experiment). c Representative Western blot showing the levels of Nup93, Nup188, Nup205, Nup98, EZH2, Suz12, CTCF, EED upon Nup93, Nup188 and Nup205 Kd (data from a single experiment). d Representative Western blot showing siRNAmediated knockdown of CTCF in DLD1 cells. e Epigenome Browser view of CTCF (GSM749729) (arrow indicates potential binding sites of Nup93) on HOXA gene cluster. $\mathbf{f}$ qRT-PCR analysis was used to determine mRNA levels of all HOXA genes (HOXA1 to HOXA13) upon CTCF and combined Nup93 + CTCF knockdowns in DLD1 cells. Graph represents fold change $\left(2^{\left.-\Delta \Delta C_{t}\right)}\right.$ in levels of mRNA normalized to untreated cells. Error bars SEM, data from two independent biological replicates $(N=2)$ that includes total of 6 technical replicates, $\mathrm{GLCCl}$, served as control. Nup93 Kd data (green bars) is from Fig. 3a, plotted here for comparison between Nup93 Kd with Nup93 + CTCF Kd (orange bars). Nup93 does not interact with CTCF or PRC2 complex proteins. Nup93 Kd upregulates HOXA gene expression independent of CTCF. CTCF depletion alone upregulates GLCCI1, which is unaffected upon Nup93 knockdown
\end{abstract}

expression, by knocking down CTCF alone and in combination with Nup93 (Fig. 7f). Interestingly, the depletion of CTCF alone did not affect gene expression levels of any of the genes within the HOXA gene cluster (Fig. 7f, gray bars). In contrast, CTCF depletion significantly upregulated the transcript levels of GLCCI1-a gene otherwise unaffected upon Nup93 knockdown (Fig. 7f, GLCCI1). This is consistent with the role of CTCF in regulating the organization of topologically associated domains (TADs), the disruption of which potentially impact distant genomic regions [61]. Notably, the combined knockdown of both CTCF and Nup93 upregulated expression levels of HOXA genes, comparable to Nup93 knockdown alone (Fig. 7f, compare green and orange bars). Of note, GLCCI1 showed an enhanced upregulation in Nup93 + CTCF Kd cells, suggesting an altered regulatory role for CTCF in cells subjected to a combined depletion of Nup93 and CTCF. Taken together, we conclude from these assays that Nup93 and CTCF may have complementary functions in the organization of the HOXA gene cluster.

\section{Discussion}

The regulated expression of the HOX family of transcription factors is required during early development and differentiation, whereas its untimely expression in differentiated cells is associated with disease [33, 34, 37, 62]. HOX gene expression is also maintained in differentiated cells such as human skin fibroblasts in a manner that retains their tissue-specific origin [63]. ChIP-chip studies using tiling microarrays revealed an association of Nup93 with human chromosomes 5, 7 and 16 [30]. Nup93 was enriched on the HOXA sub-cluster of human chromosome 7 [30]. Here, we show that Nup93 associates with the HOXA gene cluster in a manner dependent on its interactors Nup188 and Nup205. Furthermore, depletion of these nucleoporins showed a significant increase in the expression levels of HOXA genes. This was consistent with a disengagement of the HOXA gene locus from the nuclear periphery in Nup93-/Nup188-/Nup205-depleted cells. In addition, the upregulation of HOXA genes upon Nup93 depletion was associated with an increase in active histone marks, reduced inactive marks and enrichment of a transcription elongation mark.

\section{Implications of the association of nucleoporins with chromatin}

Several studies across organisms have consistently shown an association of the mobile nucleoporins such as Nup98, Nup50 and Nup153 with chromatin in addition to regulating nuclear transport $[3,6,9,13,15,22,23]$. We corroborated previous findings of Brown et al. [30] and show that Nup93 indeed associates with the promoters of HOXA1, HOXA 3 and HOXA5 and represses HOXA gene expression (Fig. $3 \mathrm{a}-\mathrm{C}$ ). It is conceivable that the repressive mechanism of Nup93 could potentially extend to other HOX gene clusters such as the HOXB, HOXC and HOXD, respectively. Chromatin conformation capture assays (such as $5 \mathrm{C}$ ) have shown that the silenced HOXA gene cluster adopts a folded loop structure in a human myeloid leukemia cell line THP1 [64]. We speculate that nucleoporins such as Nup93 may further modulate the local three-dimensional organization of the topologically associated domains (TADs) within the HOX gene cluster [65]. Our studies provide evidence to the growing body of literature which reinforce the role of nucleoporins in regulating chromatin organization and gene expression. Gene expression regulation is typically accompanied by an altered occupancy of active and inactive histone marks on gene promoters $[66,67]$. The active state of the HOXA gene cluster is marked by active histone marks such as $\mathrm{H} 3 \mathrm{~K} 9 \mathrm{ac}$ and H3K4me3, while the inactive state shows an enrichment of inactive marks such as $\mathrm{H} 3 \mathrm{~K} 9 \mathrm{me} 3$ and H3K27me3 [36, 66, 68]. For instance, histone deacetylases and PRC2 complex proteins modify levels of active and inactive histone marks on the HOXA gene cluster in NT2/D1 embryonal carcinoma cells [38]. Interestingly, a recent Dam-ID study showed that Nup93 associates with chromatin at the nuclear periphery [69]. Considering the localization of the HOXA gene cluster on the gene 
poor chromosome 7 territory, proximal to the nuclear periphery, we found a sequestration of the HOXA gene cluster to the nuclear periphery potentially mediated by the Nup93 sub-complex but not Nup98 (Fig. 4). We surmise that the depletion of Nup93, Nup188 or Nup205 and their reduced stability, enhances the accessibility of the HOXA gene cluster to transcriptional activators and epigenetic modulators that could facilitate their untimely expression of HOXA genes-the physiological ramifications of which remain unclear.

\section{Role of nucleoporins in nuclear transport and chromatin organization}

Nucleoporins regulate nuclear import and export of mRNA, RNA and proteins [1]. In addition, an increasing number of evidences implicate nucleoporins in gene regulation [4, 69-75]. Furthermore, the composition of the nuclear pore complex (NPC) is variable across cells types, which interestingly has limited effect on nuclear transport [14]. In embryonic stem cells, Nup210 is absent but is specifically incorporated into the NPC during differentiation [14]. Dam-ID studies reveal that Nup153 regulates expression of cell identity genes independent of its role in nuclear transport [69]. Nup98 is involved in both nucleocytoplasmic transport and gene regulation $[3,13$, $76,77]$, since Nup98 interacts with the mRNA export factor Rae1 and regulates mRNA export [57]. Nup98 associates with developmentally active genes such as GRIK1, ERBB4, NRG1 and DCC and regulates their expression levels during differentiation [13]. The Nup98-HOXA9 fusion protein associates with and inappropriately activates the HOX gene cluster in mouse embryonic stem cells in a manner dependent on the Crm1 protein [78]. Interestingly, Nup98 depletion in DLD1 cells did not alter either the spatial localization or the expression levels of the HOXA gene (Fig. 4 and Fig. S2b), notwithstanding its impact on nuclear transport (Fig. 6). This suggests an independent role for Nup98 in regulating nuclear transport but not HOXA gene expression. However, in cells depleted of Nup93, Nup188 or Nup205, nuclear export was relatively unaffected although nuclear import was reduced (Fig. 6a). Taken together, these findings implicate nucleoporins such as Nup93, Nup188 and Nup205 as modulators of chromatin organization in addition to their nuclear transport functions.

\section{Potential mechanisms of nucleoporin-chromatin interactions}

The mechanisms by which core nucleoporins associate with DNA are unclear. More importantly, several findings suggest that nucleoporins are involved in chromatin remodeling owing to their association with chromatin modifiers such as the SAGA complex, HDACs,
RSC complex, SUMO proteases, SENP1, SENP2 and MSL complex [23, 77, 79-82]. Chromatin remodeling complexes such as the SAGA complex-a transcriptional activator, associates with the nuclear pore complex and activates HXK1, INO1 and GAL genes when recruited to the NPC [5, 10, 83-87]. Nup2, Nup60, Nic96, Nup116, Mlp1 and Mlp2 are enriched on transcriptionally active regions in S. cerevisiae [85, 88]. Furthermore, ARP6 links the active housekeeping gene RPP1A, involved in ribosome biogenesis to the nuclear pore complex [89]. Nup170p represses ribosomal biogenesis genes and genes on the sub-telomeric region [23, 89]. Nup120 and Nup133 also core nucleoporins repress SUC2 gene expression in yeast [90]. Interestingly, Nup93 tethers and regulates the expression of cell identity genes, predominantly localized at the nuclear periphery [69]. The tethering of HOXA gene cluster to the nuclear periphery and its repression by the Nup93 sub-complex adds to the repertoire of nucleoporin-mediated gene repression events (Fig. 4). Analyses of protein-protein interaction networks using BIOGRID [91] of human Nup93 shows that Nup93 interacts with chromatin modifiers such as HDAC11, HDAC9, HDAC5 and PCR2 complex proteins-EED and Suz12. It is conceivable that Nup93 and its interactors associate with transcriptional repressors in repressing the HOXA gene cluster, although we did not detect a direct association between Nup93 and the chromatin repressive complex (PRC2) (Fig. 7b). ChIP-mass spectrometric approaches may identify putative interactors of Nup93 involved in chromatin organization.

\section{Nucleoporins as repressors of HOXA gene expression independent of CTCF}

Regulation of HOXA gene expression is essential during early development, since the aberrant expression of HOX genes leads to developmental defects [92-94]. Active HOX genes cluster into transcriptionally active domains as shown using chromatin conformation capture analyses in mouse embryonic tissues [95]. Similarly, HOXA gene regulation is also important in adult tissues since their aberrant expression is associated with various cancers [34]. Furthermore, CTCF is an important regulator of the 3D organization and silencing of the HOXA gene cluster [40]. Notably, CTCF is associated closer to the 5 ' region of the HOX gene cluster, in a manner that does not overlap with Nup93 binding sites, suggesting the complementary and potentially independent roles of Nup93 and CTCF in the maintenance of HOXA gene repression. However, it is unclear if CTCF silences HOXA gene cluster in differentiated cells by recruiting regulatory proteins such as PRC2. We showed that Nup93 depletion in DLD1 cells did not alter the levels of CTCF or PRC2 complex proteins-EZH2, Suz12 and EED (Fig. 7c). We surmise 
that CTCF or PRC2 proteins may have altered chromatin occupancy in the absence of Nup93 sub-complex, which remains to be elucidated by ChIP-sequencing of CTCF or PRC2 complex proteins in the absence of Nup93 [38]. This is further consistent with the significant upregulation of the GLCCI1 gene in cells depleted of both Nup93 and CTCF as compared CTCF-depleted cells (Fig. 7f).

We speculate a novel role for Nup93 and its interactors in regulating chromatin compaction of the HOXA gene cluster in a mechanism that curtails the aberrant expression of HOXA genes. Our studies open up challenging new frontiers for identifying the structural and molecular mechanisms of nucleoporin-mediated chromatin organization and function in paradigms of development, differentiation and disease.

\section{Conclusions}

Our studies unravel a novel role for nucleoporins Nup93 and its interactors Nup188 and Nup205 in mediating the repression and tethering of the HOXA gene cluster to the nuclear periphery in diploid DLD1 cells. Depletion of Nup93, Nup188 or Nup205 significantly enhances HOXA gene expression. The elevated levels of HOXA gene expression upon the depletion of Nup93 or its interactors-Nup188 and Nup205, is associated with an increase in the occupancy of active histone marks, and decreased levels of inactive histone marks with a concomitant increase in transcriptional elongation marks within the HOXA gene.

\section{Methods}

\section{Cell culture}

Colorectal adenocarcinoma cell line-DLD1, was a gift from the laboratory of Thomas Ried. DLD1 cells were cultured in RPMI media (Invitrogen, 11875), supplemented with $10 \%$ fetal bovine serum (FBS, Invitrogen, 6140-079, Carlsbad, USA), $100 \mathrm{U} / \mathrm{ml}$ penicillin $/ 100 \mu \mathrm{g} / \mathrm{ml}$ of streptomycin at $37{ }^{\circ} \mathrm{C}$ with $5 \% \mathrm{CO}_{2}$. The authenticity of DLD1 cells was validated independently by karyotyping (data not shown).

\section{Transient siRNA-mediated knockdown and overexpression}

Transient knockdowns were performed using siRNA oligonucleotides from Dharmacon, USA. Briefly, DLD1 cells $\left(\sim 0.2 \times 10^{6}\right)$ were plated in individual wells of a six-well plate, $24 \mathrm{~h}$ prior to transfection for cells to attain a confluency of $\sim 50-60 \%$. The cells were transfected with siRNA oligonucleotides $(50 \mathrm{nM})$ using RNAiMax (Invitrogen). After $48 \mathrm{~h}$ of knockdown, cells were processed for Western blots or RNA extraction. For recovery experiments, cells were allowed to recover in culture for 8 days after 48 h of Nup93, Nup188 and Nup205 depletion. Nup93 knockdown was performed by using a combination of oligo-1 (5'GCGCTAATTTA CTACTGCA3') and oligo-2 (5'AGAGTGAAGTGGCG GACAA3 $\left.3^{\prime}\right)-25 \mathrm{nM}$ each. Two independent siRNA oligos against Nup188, Nup205 and Nup98 were tested for their knockdown efficiency. Nup188 oligo-1 (5'GGUAG UAGGCAGACCAAUAUU3'), oligo_2 (5'GCCTTTCTG CGCTTGATCACCACCC3'); Nup205 oligo-1 (5'GGAA UUAAUCCCAGAACUAUU3'), oligo2-(5'AGAUGGUG AAGGAGGAAUAUU3'); Nup98 oligo-1 (5'GTGAAG GGCTAAATAGGAA3'), oligo-2 (5'TGTCAGACCCT AAGAAGAA3'). CTCF knockdown was performed using a single oligo (5'CAAGAAGCGGAGAGGACGA3') at a final concentration of $50 \mathrm{nM}$. ON-TARGETplus non-targeting siRNA (siNeg, Dharmacon) was used as a negative control.

For overexpression experiments, cells were first transfected with siRNA against Nup188 and Nup205 using Lipofectamine RNAi-Max reagent (Invitrogen). After $24 \mathrm{~h}$, cells were washed with DPBS and transfected with full-length Nup93 (Nup93-pEGFP-N1) using Mirus2020 reagent (cat. MIR5400). After 48 h of Nup93-GPF transfection, cells were either fixed with 4\% paraformaldehyde or harvested for RNA extraction using Trizol. Nup93GFP construct was a kind gift from Radha Chauhan.

\section{Protein extraction and immunoblotting}

Cells were lysed in RIPA buffer $(50 \mathrm{mM}$ Tris-Cl $\mathrm{pH}$ 7.4, $150 \mathrm{mM} \mathrm{NaCl}, 0.1 \%$ SDS, $0.1 \%$ sodium azide, $0.5 \%$ sodium deoxycholate, $1 \mathrm{mM}$ EDTA, $1 \% \mathrm{NP}-40,1 \times$ protease inhibitor cocktail) and centrifuged at $13,000 \mathrm{~g}$ for $10 \mathrm{~min}$ at $4{ }^{\circ} \mathrm{C}$. The supernatant was separated and used for protein estimation using BCA kit (cat no. 23225). Total protein $(20 \mu \mathrm{g}$, estimated to be within the linear range of detection) was used for each sample preparation. Samples were lysed in $1 \times$ Laemmli buffer (Tris$\mathrm{HCl} \mathrm{pH} \mathrm{6.8,2 \%} \mathrm{SDS,} \mathrm{20 \%} \mathrm{glycerol,} 0.2 \%$ bromophenol blue, $0.025 \% \beta$-mercaptoethanol) and denatured at $95{ }^{\circ} \mathrm{C}$ for $5 \mathrm{~min}$. Protein samples were resolved by SDS-PAGE and were transferred to activated polyvinylidene fluoride membrane (PVDF, Millipore, cat no. IPVH00010), followed by blocking with $5 \%$ nonfat-dried skim milk/1× TBST (Tris-buffer saline, $0.1 \%$ Tween 20) for $1 \mathrm{~h}$ at RT. Primary antibodies were diluted in $0.5 \%$ milk $/ 1 \times$ TBST buffer. All antibody dilutions are within the linear range of detection. Rabbit anti-Nup93 (1:500, sc-292099, LotE0211, Santa Cruz Biotechnology, CA), rabbit antiNup188 (1:1000, Abcam, ab86601, Lot-GR43443-4), mouse anti-Nup98 (1:500, sc-74553, Lot-H0108, Santa Cruz Biotechnology, Santa Cruz, CA), rabbit antiNup205 antibody (1:500, HPA024574, Lot-R11937, Atlas Antibodies), rabbit anti-EED (1:500, ab4469, Lot-GR51357-1), rabbit anti-EZH2 (1:500, ab3748, Lot-GR252135-1), rabbit anti-Suz12 (1:500, ab12073, 
Lot-GR79631-1) and rabbit anti-CTCF antibody (1:500, 07-729, Lot-2375606, Millipore). Secondary antibodies: donkey anti-rabbit immunoglobulin $\mathrm{G}$ horseradish peroxidase $(1: 10,000$, GE NA9340V) and sheep anti-mouse immunoglobulin G-HRP (1: 10,000, NA9310V) were diluted in $0.5 \%$ milk $(1 \times$ TBST). The blots were developed using enhanced chemiluminescence detection reagents (ECL Prime, 89168-782) at incremental exposures of $10 \mathrm{~s}$ acquired under a chemiluminescence system LAS4000 (GE). Densitometry analysis of Western blots was done using ImageJ software from three independent biological replicates. GAPDH was used as internal control for normalization.

\section{Reverse transcription-PCR and real-time quantitative PCR} Cells were washed with $1 \mathrm{X}$ PBS, and total RNA was extracted using Trizol method [96]; cDNA was synthesized from total RNA with the ImProm-II reverse transcription system using Oligo(dT) primers (Promega, A3800); cDNA was used as a template and RT-PCR was carried out using intron-exon junction primers (Additional file 5: Table S1). $\beta$-actin and glyceraldehyde-3-phosphate dehydrogenase (GAPDH) were used as internal controls. The template cDNA was serially diluted to optimize the extent of amplification in the linear range. Realtime quantitative PCR was performed using Bio-Rad RT-PCR instrument (CFX96 Touch) in $10 \mu$ of reaction mixtures containing KAPA SYBR Green RT-PCR mix and $2 \mu \mathrm{M}$ each of the forward and reverse primer, respectively (Additional file 5: Table S2). Fold change was calculated by double normalization of $\mathrm{Ct}$ values to the internal control and untreated samples by $2^{-\Delta \Delta C_{t}}$ method [97].

\section{Immunoprecipitation (IP) and Co-IP}

Cells were lysed using IP lysis buffer (50 mM HEPES$\mathrm{pH}$ 8.0, $140 \mathrm{mM} \mathrm{NaCl}, 1 \% \mathrm{NP}-40,0.1 \%$ sodium deoxycholate, $0.1 \%$ SDS and $1 \mathrm{mM}$ EDTA) in the presence of $1 \mathrm{X}$ complete protease inhibitor cocktail (PIC). Lysates were pre-cleared using protein A dynabeads (Invitrogen, 10002D), $1 \mathrm{~h}$ at $4{ }^{\circ} \mathrm{C}$. Pre-cleared extracts were incubated overnight at $4{ }^{\circ} \mathrm{C}$ with anti-Nup93, anti-Nup205 and anti-Nup 188 antibodies independently $(2 \mu \mathrm{g} / 500 \mu \mathrm{g}$ of total protein). IP complexes were captured using protein A dynabeads (pre-blocked with $0.5 \%$ BSA/1X PBS) and washed with lysis buffer and high salt wash buffer-1 (500 $\mathrm{mM} \mathrm{NaCl}$ in lysis buffer), and wash buffer-2 (20 mM Tris-HCL (pH 8.0), 1 mM EDTA, 0.5\% NP-40 and 50× PIC). Elution was performed using Laemmli loading buffer and analyzed by Western blotting. For Co-IP, cells were lysed in Co-IP buffer $(50 \mathrm{mM}$ Tris-HCl, $\mathrm{pH} 8.0$, $150 \mathrm{mM} \mathrm{NaCl}$ and $0.5 \% \mathrm{NP}-40$ ) supplemented with protease inhibitor cocktail. Co-IP washes were performed in the same Co-IP lysis buffer.

\section{Chromatin immunoprecipitation}

DLD1 cells $\left(\sim 1.0 \times 10^{7}\right)$ were cross-linked using $1 \%$ formaldehyde for $10 \mathrm{~min}$ at RT. Cross-linking was quenched using $150 \mathrm{mM}$ glycine, and cells were lysed in $1 \mathrm{ml}$ swelling buffer (25 mM HEPES pH 8.0, $1.5 \mathrm{mM} \mathrm{MgCl}_{2}, 10 \mathrm{mM}$ $\mathrm{KCl}, 0.1 \% \mathrm{NP}-40,1 \mathrm{X} \mathrm{PIC}$ ), and nuclei were recovered by centrifugation at $2000 \mathrm{rpm}$. Fixed nuclei were resuspended in $1 \mathrm{ml}$ sonication buffer (50 mM HEPES $\mathrm{pH}$ 8.0, $140 \mathrm{mM} \mathrm{NaCl}, 1 \mathrm{mM}$ EDTA, 1\% Triton-X-100, 0.1\% sodium deoxycholate and $0.1 \%$ SDS) supplemented with protease inhibitor cocktail and sonicated using Bioruptor Twin sonicator (Diagenode) to generate fragment sizes of 100-500 bp. Sonicated chromatin was separated by centrifugation at $13,000 \mathrm{~g}$ at $4{ }^{\circ} \mathrm{C}$ for $10 \mathrm{~min}$. Supernatant was pre-cleared using protein A dynabeads (Invitrogen), $1 \mathrm{~h}$ at $4{ }^{\circ} \mathrm{C}$. The amount of DNA was estimated using NanoDrop 2000. Nup93 antibody validated according to ENCODE guidelines $(\sim 2 \mu \mathrm{g})$ was added to $\sim 100 \mu \mathrm{g}$ of chromatin sample and diluted to $\sim 1 \mathrm{ml}$ in sonication buffer and incubated overnight at $4{ }^{\circ} \mathrm{C}$ [98]. IP complexes were captured using protein A dynabeads (pre-blocked with $0.5 \% \mathrm{BSA} / 1 \mathrm{X} \mathrm{PBS}$ ) and washed three times (at $4{ }^{\circ} \mathrm{C}$, $11 \mathrm{rpm}$ on end-to-end rotor) each with sonication buffer, wash buffer-1 (50 mM HEPES pH 8.0, $500 \mathrm{mM} \mathrm{NaCl}$, $1 \mathrm{mM}$ EDTA, $1 \%$ Triton X-100, $0.1 \%$ sodium deoxycholate and $0.1 \%$ SDS), wash buffer-2 $(20 \mathrm{mM}$ Tris-HCL pH 8.0, 1 mM EDTA, 0.5\% NP-40, $250 \mathrm{mM} \mathrm{LiCl,} \mathrm{0.5 \%}$ sodium deoxycholate and $1 \times$ PIC) and TE buffer. Immunoprecipitated chromatin was eluted twice in $200 \mu \mathrm{l}$ of elution buffer (50 mM Tris-HCL pH 8.0, 1 mM EDTA, $1 \% \mathrm{SDS}, 50 \mathrm{mM} \mathrm{NaHCO}$ ) at $65^{\circ} \mathrm{C}$ for $10 \mathrm{~min}$. Input and IP fractions were treated with $20 \mu \mathrm{g}$ RNase A for $1 \mathrm{~h}$ at $42{ }^{\circ} \mathrm{C}$ followed by $40 \mu \mathrm{g}$ Proteinase $\mathrm{K}$ for $1 \mathrm{~h}$ at $65^{\circ} \mathrm{C}$. Reverse cross-linking was performed overnight at $65^{\circ} \mathrm{C}$. DNA was extracted with phenol/chloroform/isoamyl alcohol (25:24:1), ethanol precipitated using $3 \mathrm{M}$ sodium acetate ( $\mathrm{pH} 5.2)$ and $2 \mu \mathrm{g}$ of glycogen. DNA samples were washed with $70 \%$ ethanol and re-suspended in $10 \mu \mathrm{l}$ of nuclease free water. ChIP-PCR was performed using primers listed in Additional file 5: Table S1. DNA was quantified by real-time qPCR, and fold enrichment over input was calculated by $\%$ input method from $2^{-\Delta \Delta C_{\mathrm{t}}}$ [97, 99]. All ChIP-qPCR experiments were performed in two independent biological replicates as recommended in the ENCODE guidelines [100].

\section{Three-dimensional fluorescence in situ hybridization (3D-FISH)}

3D-FISH assays were performed as described previously [101]. DLD1 cells $\left(\sim 0.2 \times 10^{6}\right)$ were seeded on coverslips in a six-well plate. After $48 \mathrm{~h}$ of Nup93 knockdown, the cells were washed with ice-cold $1 \times$ PBS and treated with cytoskeletal (CSK) digestion buffer $(0.1 \mathrm{M} \mathrm{NaCl}, 0.3 \mathrm{M}$ 
sucrose, $3 \mathrm{mM} \mathrm{MgCl}, 10 \mathrm{mM}$ PIPES (pH 7.4), 0.5\% Triton X-100) for 5 min followed by fixation with 4\% PFA in $1 X$ PBS ( $\mathrm{pH} 7.4$ ) for $10 \mathrm{~min}$ at RT. The cells were permeabilized in $0.5 \%$ Triton X-100 (prepared in $1 \times$ PBS) for $10 \mathrm{~min}$ and incubated in $20 \%$ glycerol (prepared in $1 \times$ PBS) for 60 min followed by four freeze-thaw cycles in liquid nitrogen. The cells were washed three times in $1 \times$ PBS and treated with $0.1 \mathrm{~N} \mathrm{HCl}$ for $10 \mathrm{~min}$ followed by three washes in $1 \times$ PBS for $5 \mathrm{~min}$ each. The cells were incubated in $50 \%$ formamide $(\mathrm{FA}) / 2 \times$ saline sodium citrate (SSC) (pH 7.4) overnight at $4{ }^{\circ} \mathrm{C}$ or until used for hybridization. Cells were hybridized with $3 \mu \mathrm{l}$ of human whole chromosome 7 paint (Applied Spectral Imaging (ASI), Israel, or MetaSystems, USA) and nick-translated BAC DNA probe (RP11-1132K14) for HOXA gene locus $(3 \mu \mathrm{l})$. Posthybridization, coverslips were washed in $50 \% \mathrm{FA} / 2 \times \mathrm{SSC}$ (pH 7.4), thrice for $5 \mathrm{~min}$ each at $45{ }^{\circ} \mathrm{C}$, followed by three washes for $5 \mathrm{~min}$ each in $0.1 \times \mathrm{SSC}$ at $60{ }^{\circ} \mathrm{C}$. Coverslips were then counterstained with DAPI for 2 min, washed in $2 \times$ SSC and mounted in Slowfade Gold antifade (Invitrogen S36937). Image acquisition was performed on a Leica SP8 confocal microscope with a 63X Plan-Apo1.4 NA oil immersion objective using scan zoom of 2.5. Acquisition of Z-stacked images (voxel size of $0.105 \mu \mathrm{m} \times 0.105 \mu \mathrm{m}$ $\times 0.30 \mu \mathrm{m})$ was at $512 \times 512$ pixels per frame using 8-bit pixel depth for each channel. The line averaging was set to 4 , and images were collected sequentially in a three-channel mode. Distances of gene loci from the nuclear periphery were measured (in $\mu \mathrm{m}$ ) in 3D using the boundary of the DAPI signal as a marker of the nuclear periphery [50]. Briefly, surface rendering was performed for the nucleus (blue channel), HOXA gene locus (red channel), CT7 (green channel), using Huygens Professional software.

\section{Nuclear import assay}

Nuclear import assay was performed as described previously [53]. DLD1 cells $\left(\sim 0.2 \times 10^{6}\right)$ were seeded on coverslips in a six-well plate. After $24 \mathrm{~h}$ of siRNA transfection, cells were transfected with the vector encoding GR2-GFP2-M9core fusion protein. After $48 \mathrm{~h}$ of transfection, cells were treated with $5 \mu \mathrm{M}$ dexamethasone (Sigma) for $30 \mathrm{~min}$ at $37{ }^{\circ} \mathrm{C}$ and fixed with $4 \%$ paraformaldehyde. Cells were permeabilized with $0.5 \%$ Triton X-100 and immunostained with Phalloidin-Alexa 594 (Invitrogen, cat. A12381) to mark the cell boundary. Finally, cells were stained with DAPI and mounted in antifade solution. Images were acquired using Leica SP8 confocal microscopy using a $63 \times$ objective/N.A. 1.4 using 405, 488 and $594 \mathrm{~nm}$ and lasers, zoom set to 2.0. The mean fluorescence intensity of the GFP signal was determined for each cell (demarcated by phalloidin) and nucleus (demarcated by DAPI), and expressed as a ratio of the nuclear to cytoplasmic fluorescence intensity using
Image). Nuclear/cytoplasmic (N/C) fluorescence intensity ratios were calculated and plotted using GraphPad Prism software. Statistical analysis was performed using Mann-Whitney test. The GR2-GFP2-M9 construct was a kind gift from Ralph Kehlenbach.

\section{Poly (A) fluorescence in situ hybridization (FISH)}

DLD1 cells $\left(\sim 0.2 \times 10^{6}\right)$ were seeded on coverslips in a six-well plate. After $48 \mathrm{~h}$ of Nup93 knockdown, the cells were fixed with $4 \%$ PFA in $1 \times$ PBS (pH 7.4) for $15 \mathrm{~min}$ at RT. Cells were re-fixed and permeabilized with chilled methanol for $5 \mathrm{~min}$, followed by incubation in $2 \times$ SSC at RT for $10 \mathrm{~min}$. Cells were hybridized with $100 \mu \mathrm{l}$ of hybridization mix (40\% formamide, $10 \%$ dextran sulfate, $0.1 \mathrm{mg}$ salmon sperm DNA and $5 \mathrm{ng} / \mathrm{ml}$ of FAM oligo dT prepared in $2 \times \mathrm{SSC}$ solution) at $37^{\circ} \mathrm{C}$ for $3 \mathrm{~h}$. Coverslips were washed twice with $2 \times$ SSC, followed by washes with $0.1 \times$ SSC. Cells were stained with DAPI and mounted in antifade solution. Images were acquired using confocal microscopy using a $63 \times$ objective/N.A. 1.4 using 488 and $405 \mathrm{~nm}$ lasers, zoom set to 1.0. The mean fluorescence intensity of the FISH signal was determined for each cell and nucleus (demarcated by DAPI), and expressed as a ratio of the nuclear to cytoplasmic fluorescence intensity using the Cell Profiler software [102]. Nuclear/cytoplasmic $(\mathrm{N} / \mathrm{C})$ fluorescence intensity ratios were calculated and plotted using GraphPad Prism software. Statistical analysis was performed using Mann-Whitney test.

\section{Data accession from WashU Epigenome Browser}

WashU Epigenome Browser was used to visualize the openly available CTCF (GSM749729) ChIP-seq data from HeLa cells.

Antibodies used: All antibodies and their dilutions used in this study are provided in Additional file 5: Table S3.

\section{Additional files}

Additional file 1: Figure S1. a Co-Immunoprecipitation of Nup93 upon depletion of Nup205, shows reduced interaction between Nup93 and Nup188. b Nup93 ChIP PCR experiment was performed in Nup188 and Nup205 depleted cells, a representative gel image from 2 independent biological replicates. c ChIP qPCR for control PanH3 in siNeg, Nup188 and Nup205Kd cells, Y-axis represents immunoprecipitated DNA relative to $1 \%$ input ( $N=2$, independent biological replicates). d qRT-PCR analyses was performed upon Nup93, Nup188 and Nup205 depletion to check their relative transcript levels. Y-axis indicates fold change in levels of mRNA normalized to untreated cells. Error bars: S.E.M, data from a single experiment that includes 3 technical replicates. e qRT-PCR analyses upon Nup93 over expression in a background of Nup188 or Nup205 depletion as indicated. Y-axis indicates fold change in levels of mRNA normalized to untreated cells. Error bars: S.E.M, data from two biological replicates that includes 6 technical replicates. $\mathbf{f}$ Western blot quantification of Fig. 2e from 3 independent biological replicates. Y-axis: relative band intensity quantified using Image-J. g Ponceaue staining for western blot represented in Fig. 2e.

Additional file 2: Figure S2. a Two independent siRNA oligos were used to knockdown Nup93, Nup188, Nup205 and Nup98.A representative 
western blot form two independent biological replicates. GAPDH was used as an internal control. b qRT-PCR was performed for entire HOXA cluster upon Nup93, Nup188, Nup205 and Nup98 depletion using two independent siRNA oligos. Y-Axis represent fold change normalized to untreated. Data from two independent biological replicates, Error bars: S.E.M. c-e Effect of allowing cells to recover for 8 days after 48 hours of knockdown of (c) Nup93; (d) Nup188 and (e) Nup205, qRT-PCR analysis was used to determine mRNA levels of HOXA1, HOXA9 and HOXA13 genes after $48 \mathrm{~h}$ knockdown and 8 days of recovery. Error bars-S.E.M, data from one biological replicate that includes 3 technical replicates. Nup93, Nup188 and Nup205 recover to comparable levels as that of untreated cells after 8 days, along with a concomitant repression of HOXA1, HOXA9 and HOXA13 transcript levels. $\mathbf{h}-\mathbf{j}$ lgG levels in untreated, siNeg and Nup93 Kd replotted from Fig. $4 b-d$, since they are below the detection limit.

Additional file 3: Figure S3. Representative images of nuclear import assay performed using GR2-GFP2-M9 construct transfected in control (LacZ) and Nup93, Nup188, Nup205 and Nup98 depleted cells uponand +Dex (dexamethasone) treatment (to induce nuclear import of GR2-GFP2-M9 fusion protein cells were treated with $5 \mu \mathrm{M}$ dexamethasone for $30 \mathrm{~min}$ ).

Additional file 4: Figure S4. Representative images of Poly(A) RNA FISH performed using FAM labeled oligo(dT) probe (green) in Nup188 Kd and Nup205 Kd. Scale bar: $10 \mu \mathrm{m}$.

Additional file 5: Table S1. List of ChIP-qPCR primers. Table S2. List of RT-PCR primers. Table S3. Antibodies and their dilutions used in this study.

\section{Abbreviations}

Kd: knockdown; IP: immunoprecipitation; ChIP: chromatin immunoprecipitation; FISH: fluorescence in situ hybridization; HDAC: histone deacetylase; PRC: Polycomb repressive complex; $\mathrm{QPCR}$ : quantitative polymerase chain reaction; H3K9ac: histone H3 lysine 9 acetylation; H3K27me3: histone H3 lysine 27 tri-methylation; H3K36me3: histone H3 lysine 36 tri-methylation; ENCODE: encyclopedia of DNA elements.

\section{Authors' contributions}

$\mathrm{AL}, \mathrm{KK}, \mathrm{KS}$ designed the experiments, analyzed the data and wrote the manuscript. AL performed the experiments. All authors read and approved the final manuscript.

\section{Acknowledgements}

We gratefully acknowledge IISER, Pune for facilities, intramural funding of IISER Pune and extramural funding through an intermediate fellowship to KS from Wellcome Trust-DBT India Alliance (Award No. 500164/Z/09/Z). We thank Chromosome Biology Lab (CBL) members for their critical inputs. We thank Sanjeev Galande and his laboratory members for reagents and suggestions. We are grateful to Jomon Joseph for reagents and suggestions. We thank Girish Deshpande for his critical comments on the manuscript. We thank Ralph Kehlenbach and Radha Chauhan for the GR2-GFP2-M9 and Nup93 overexpression constructs, respectively.

\section{Competing interests}

The authors declare that they have no competing interests.

\section{Availability of supporting data}

All data generated or analyzed during this study are included in this article.

\section{Funding}

Intramural funding of IISER-Pune and extramural funding through an intermediate fellowship to KS from Wellcome Trust-DBT India Alliance (Award No. 500164/Z/09/Z).

Received: 20 August 2016 Accepted: 23 November 2016 Published online: 03 December 2016

\section{References}

1. Wente SR, Rout MP. The nuclear pore complex and nuclear transport. Cold Spring Harb Perspect Biol. 2010;2:a000562.

2. Wälde S, Kehlenbach RH. The part and the whole: functions of nucleoporins in nucleocytoplasmic transport. Trends Cell Biol. 2010;20:461-9.

3. Capelson M, Liang Y, Schulte R, Mair W, Wagner U, Hetzer MW. Chromatin-bound nuclear pore components regulate gene expression in higher eukaryotes. Cell. 2010;140:372-83.

4. Kalverda B, Pickersgill H, Shloma WV, Fornerod M. Nucleoporins directly stimulate expression of developmental and cell-cycle genes inside the nucleoplasm. Cell. 2010;140:360-71.

5. Taddei A, Van Houwe G, Hediger F, Kalck V, Cubizolles F, Schober H, Gasser SM. Nuclear pore association confers optimal expression levels for an inducible yeast gene. Nature. 2006;441:774-8.

6. Jacinto FV, Benner C, Hetzer MW. The nucleoporin Nup153 regulates embryonic stem cell pluripotency through gene silencing. Genes Dev. 2015;29:1224-38.

7. Brown CR, Silver PA. Transcriptional regulation at the nuclear pore complex. Curr Opin Genet Dev. 2007;17:100-6.

8. Light WH, Brickner JH. Nuclear pore proteins regulate chromatin structure and transcriptional memory by a conserved mechanism. Nucleus. 2013:4:357-60.

9. Light WH, Freaney J, Sood V, Thompson A, D'Urso A, Horvath CM, Brickner JH. A conserved role for human Nup98 in altering chromatin structure and promoting epigenetic transcriptional memory. PLoS Biol. 2013;11:e1001524.

10. Light WH, Brickner DG, Brand VR, Brickner JH. Interaction of a DNA zip code with the nuclear pore complex promotes $\mathrm{H} 2 \mathrm{~A}$.Z incorporation and INO1 transcriptional memory. Mol Cell. 2010;40:112-25.

11. Kalverda B, Fornerod M. Characterization of genome-nucleoporin interactions in Drosophila links chromatin insulators to the nuclear pore complex. Cell Cycle. 2010;9:4812-7.

12. Ishii K, Arib G, Lin C, Van Houwe G, Laemmli UK. Chromatin boundaries in budding yeast: the nuclear pore connection. Cell. 2002;109:551-62.

13. Liang Y, Franks TM, Marchetto MC, Gage FH, Hetzer MW. Dynamic association of NUP98 with the human genome. PLoS Genet. 2013;9:e1003308.

14. D’Angelo MA, Gomez-Cavazos JS, Mei A, Lackner DH, Hetzer MW. A change in nuclear pore complex composition regulates cell differentiation. Dev Cell. 2012;22:446-58.

15. Buchwalter AL, Liang $Y$, Hetzer MW. Nup50 is required for cell differentiation and exhibits transcription-dependent dynamics. Mol Biol Cell. 2014;25:2472-84.

16. Palancade B, Liu X, Garcia-Rubio M, Aguilera A, Zhao X, Doye V. Nucleoporins prevent DNA damage accumulation by modulating Ulp1dependent sumoylation processes. Mol Biol Cell. 2007;18:2912-23.

17. Khadaroo B, Teixeira MT, Luciano P, Eckert-Boulet N, Germann SM, Simon MN, Gallina I, Abdallah P, Gilson E, Géli V, Lisby M. The DNA damage response at eroded telomeres and tethering to the nuclear pore complex. Nat Cell Biol. 2009;11:980-7.

18. Breuer $\mathrm{M}$, Ohkura $\mathrm{H}$. A negative loop within the nuclear pore complex controls global chromatin organization. Genes Dev. 2015;29:1789-94.

19. Strambio-De-Castillia C, Niepel M, Rout MP. The nuclear pore complex: bridging nuclear transport and gene regulation. Nat Rev Mol Cell Biol. 2010;11:490-501.

20. Griffis E. Nup98 is a mobile nucleoporin with transcription-dependent dynamics. Mol Biol Cell. 2002;13:1282-97.

21. Hou C, Corces VG. Nups take leave of the nuclear envelope to regulate transcription. Cell. 2010;140:306-8.

22. Vaquerizas JM, Suyama R, Kind J, Miura K, Luscombe NM, Akhtar A. Nuclear pore proteins nup153 and megator define transcriptionally active regions in the Drosophila genome. PLoS Genet. 2010;6:e1000846.

23. Van de Vosse DW, Wan Y, Lapetina DL, Chen WM, Chiang JH, Aitchison JD, Wozniak RW. A role for the nucleoporin Nup170p in chromatin structure and gene silencing. Cell. 2013;152:969-83.

24. Vollmer B, Antonin W. The diverse roles of the Nup93/Nic96 complex proteins - structural scaffolds of the nuclear pore complex with additional cellular functions. Biol Chem. 2014;395:515-28.

25. Sachdev R, Sieverding C, Flötenmeyer M, Antonin W. The C-terminal domain of Nup93 is essential for assembly of the structural backbone of nuclear pore complexes. Mol Biol Cell. 2012;23:740-9. 
26. Grandi P, Dang T, Pané N, Shevchenko A, Mann M, Forbes D, Hurt E. Nup93, a vertebrate homologue of yeast Nic96p, forms a complex with a novel 205-kDa protein and is required for correct nuclear pore assembly. Mol Biol Cell. 1997;8:2017-38.

27. Miller BR, Powers M, Park M, Fischer W, Forbes DJ. Identification of a new vertebrate nucleoporin, Nup188, with the use of a novel organelle trap assay. Mol Biol Cell. 2000;11:3381-96.

28. Kosinski J, Mosalaganti S, von Appen A, Teimer R, DiGuilio AL, Wan W, Bui KH, Hagen WJ, Briggs JA, Glavy JS, Hurt E, Beck M. Molecular architecture of the inner ring scaffold of the human nuclear pore complex. Science. 2016:352:363-5.

29. Rabut G, Doye V, Ellenberg J. Mapping the dynamic organization of the nuclear pore complex inside single living cells. Nat Cell Biol. 2004;6:1114-21.

30. Brown CR, Kennedy CJ, Delmar VA, Forbes DJ, Silver PA. Global histone acetylation induces functional genomic reorganization at mammalian nuclear pore complexes. Genes Dev. 2008;22:627-39.

31. Rousseau M, Crutchley JL, Miura H, Suderman M, Blanchette M, Dostie J. Hox in motion: tracking HoxA cluster conformation during differentiation. Nucleic Acids Res. 2014;42:1524-40.

32. Makiyama K, Hamada J, Takada M, Murakawa K, Takahashi Y, Tada M, Tamoto E, Shindo G, Matsunaga A, Teramoto K, Komuro K, Kondo S, Katoh H, Koike T, Moriuchi T. Aberrant expression of HOX genes in human invasive breast carcinoma. Oncol Rep. 2005;13:673-9.

33. Maeda K, Hamada J, Takahashi Y, Tada M, Yamamoto Y, Sugihara T, Moriuchi T. Altered expressions of HOX genes in human cutaneous malignant melanoma. Int J Cancer. 2005;114:436-41.

34. Bhatlekar S, Fields JZ, Boman BM. HOX genes and their role in the development of human cancers. J Mol Med. 2014;92:811-23.

35. Mustafa M, Lee JY, Kim MH. CTCF negatively regulates HOXA10 expression in breast cancer cells. Biochem Biophys Res Commun. 2015;467:828-34.

36. Novak P, Jensen T, Oshiro MM, Wozniak RJ, Nouzova M, Watts GS, Klimecki WT, Kim C, Futscher BW. Epigenetic inactivation of the HOXA gene cluster in breast cancer. Cancer Res. 2006;66:10664-70.

37. Bitu CC, Destro MF, Carrera M, da Silva SD, Graner E, Kowalski LP, Soares FA, Coletta RD. HOXA1 is overexpressed in oral squamous cell carcinomas and its expression is correlated with poor prognosis. BMC Cancer. 2012;12:146.

38. Xu M, Zhao GN, Lv X, Liu G, Wang LY, Hao DL, Wang J, Liu DP, Liang CC. CTCF controls HOXA cluster silencing and mediates PRC2-repressive higher-order chromatin structure in NT2/D1 cells. Mol Cell Biol. 2014;34:3867-79.

39. Narendra V, Rocha PP, An D, Raviram R, Skok JA, Mazzoni EO, Reinberg D. CTCF establishes discrete functional chromatin domains at the Hox clusters during differentiation. Science. 2015;347:1017-21.

40. Ferraiuolo MA, Rousseau M, Miyamoto C, Shenker S, Wang XQ, Nadler M, Blanchette M, Dostie J. The three-dimensional architecture of Hox cluster silencing. Nucleic Acids Res. 2010;38:7472-84.

41. Bermejo R, Kumar A, Foiani M. Preserving the genome by regulating chromatin association with the nuclear envelope. Trends Cell Biol. 2012;22:465-73.

42. Sexton T, Schober H, Fraser P, Gasser SM. Gene regulation through nuclear organization. Nat Struct Mol Biol. 2007;14:1049-55.

43. Zimber A, Nguyen QD, Gespach C. Nuclear bodies and compartments: functional roles and cellular signalling in health and disease. Cell Signal. 2004:16:1085-104.

44. Zuleger N, Robson MI, Schirmer EC. The nuclear envelope as a chromatin organizer. Nucleus. 2011;2:339-49.

45. Sengupta K, Upender MB, Barenboim-Stapleton L, Nguyen QT, Wincovitch SM, Garfield SH, Difilippantonio MJ, Ried T. Artificially introduced aneuploid chromosomes assume a conserved position in colon cancer cells. PLoS ONE. 2007;2:e199.

46. Theerthagiri G, Eisenhardt N, Schwarz H, Antonin W. The nucleoporin Nup188 controls passage of membrane proteins across the nuclear pore complex. J Cell Biol. 2010;189:1129-42.

47. Braun DA, Sadowski CE, Kohl S, Lovric S, Astrinidis SA, Pabst WL, Gee HY, Ashraf S, Lawson JA, Shril S, Airik M, Tan W, Schapiro D, Rao J, Choi WI, Hermle T, Kemper MJ, Pohl M, Ozaltin F, Konrad M, Bogdanovic R, Büscher R, Helmchen U, Serdaroglu E, Lifton RP, Antonin W, Hildebrandt
F. Mutations in nuclear pore genes NUP93, NUP205 and XPO5 cause steroid-resistant nephrotic syndrome. Nat Genet. 2016;48:457-65.

48. Von Appen A, Kosinski J, Sparks L, Ori A, DiGuilio AL, Vollmer B, Mackmull MT, Banterle N, Parca L, Kastritis P, Buczak K, Mosalaganti S, Hagen W, Andres-Pons A, Lemke EA, Bork P, Antonin W, Glavy JS, Bui KH, Beck M. In situ structural analysis of the human nuclear pore complex. Nature. 2015:526:140-3.

49. Finlan LE, Sproul D, Thomson I, Boyle S, Kerr E, Perry P, Ylstra B, Chubb $J$ R, Bickmore WA. Recruitment to the nuclear periphery can alter expression of genes in human cells. PLoS Genet. 2008;4:e1000039.

50. Shachar S, Voss TC, Pegoraro G, Sciascia N, Misteli T. Identification of gene positioning factors using high-throughput imaging mapping Cell. 2015;162:911-23.

51. Sakamoto $Y$, Watanabe $S$, Ichimura T, Kawasuji M, Koseki H, Baba H, Nakao M. Overlapping roles of the methylated DNA-binding protein MBD1 and polycomb group proteins in transcriptional repression of HOXA genes and heterochromatin foci formation. J Biol Chem. 2007:282:16391-400.

52. Sims RJ, Reinberg D. Processing the H3K36me3 signature. Nat Genet. 2009:41:270-1.

53. Hutten S, Wälde S, Spillner C, Hauber J, Kehlenbach RH. The nuclear pore component Nup358 promotes transportin-dependent nuclear import. J Cell Sci. 2009;122(Pt 8):1100-10.

54. Chakraborty P, Satterly N, Fontoura BM. Nuclear export assays for poly(A) RNAs. Methods. 2006;39:363-9.

55. Powers MA, Forbes DJ, Dahlberg JE, Lund E. The vertebrate GLFG nucleoporin, Nup98, is an essential component of multiple RNA export pathways. J Cell Biol. 1997;136:241-50.

56. Fabre E, Boelens WC, Wimmer C, Mattaj IW, Hurt EC. Nup145p is required for nuclear export of mRNA and binds homopolymeric RNA in vitro via a novel conserved motif. Cell. 1994;78:275-89.

57. Pritchard CE, Fornerod M, Kasper LH, van Deursen JM. RAE1 is a shuttling mRNA export factor that binds to a GLEBS-like NUP98 motif at the nuclear pore complex through multiple domains. J Cell Biol. 1999;145:237-54

58. Li X, Isono K, Yamada D, Endo TA, Endoh M, Shinga J, Mizutani-Koseki Y, Otte AP, Casanova M, Kitamura H, Kamijo T, Sharif J, Ohara O, Toyada T, Bernstein BE, Brockdorff N, Koseki H. Mammalian polycomb-like Pcl2/ Mtf2 is a novel regulatory component of PRC2 that can differentially modulate polycomb activity both at the Hox gene cluster and at Cdkn2a genes. Mol Cell Biol. 2011;31:351-64.

59. Peña-Hernández R, Marques M, Hilmi K, Zhao T, Saad A, Alaoui-Jamali MA, del Rincon SV, Ashworth T, Roy AL, Emerson BM, Witcher M. Genome-wide targeting of the epigenetic regulatory protein CTCF to gene promoters by the transcription factor TFII-I. Proc Natl Acad Sci USA. 2015;112:E677-86

60. Vieux-Rochas M, Fabre PJ, Leleu M, Duboule D, Noordermeer D. Clustering of mammalian Hox genes with other H3K27me3 targets within an active nuclear domain. Proc Natl Acad Sci USA. 2015:112:4672-7.

61. Lupiáñez DG, Kraft K, Heinrich V, Krawitz P, Brancati F, Klopocki E, Horn D, Kayserili H, Opitz JM, Laxova R, Santos-Simarro F, Gilbert-Dussardier B, Wittler L, Borschiwer M, Haas SA, Osterwalder M, Franke M, Timmermann B, Hecht J, Spielmann M, Visel A, Mundlos S. Disruptions of topological chromatin domains cause pathogenic rewiring of geneenhancer interactions. Cell. 2015:161:1012-25.

62. Calvo R, West J, Franklin W, Erickson P, Bemis L, Li E, Helfrich B, Bunn P, Roche J, Brambilla E, Rosell R, Gemmill RM, Drabkin HA. Altered HOX and WNT7A expression in human lung cancer. Proc Natl Acad Sci USA 2000;97:12776-81.

63. Chang HY, Chi JT, Dudoit S, Bondre C, van de Rijn M, Botstein D, Brown PO. Diversity, topographic differentiation, and positional memory in human fibroblasts. Proc Natl Acad Sci USA. 2002;99:12877-82.

64. Fraser J, Rousseau M, Shenker S, Ferraiuolo MA, Hayashizaki Y, Blanchette M, Dostie J. Chromatin conformation signatures of cellular differentiation. Genome Biol. 2009;10:R37.

65. Noordermeer D, Leleu M, Schorderet P, Joye E, Chabaud F, Duboule D. Temporal dynamics and developmental memory of 3D chromatin architecture at Hox gene loci. elife. 2014;3:e02557.

66. Atkinson SP, Koch CM, Clelland GK, Willcox S, Fowler JC, Stewart R, Lako M, Dunham I, Armstrong L. Epigenetic marking prepares the human 
HOXA cluster for activation during differentiation of pluripotent cells. Stem Cells. 2008;26:1174-85.

67. Gonzalez-Sandoval A, Gasser SM. On TADs and LADs: spatial control over gene expression. Trends Genet. 2016;32:485-95.

68. Srivastava S, Dhawan J, Mishra RK. Epigenetic mechanisms and boundaries in the regulation of mammalian Hox clusters. Mech Dev. 2015;138(Pt 2):160-9.

69. Ibarra A, Benner C, Tyagi S, Cool J, Hetzer MW. Nucleoporin-mediated regulation of cell identity genes. Genes Dev. 2016.

70. Liang Y, Hetzer MW. Functional interactions between nucleoporins and chromatin. Curr Opin Cell Biol. 2011:23:65-70.

71. Ibarra A, Hetzer MW. Nuclear pore proteins and the control of genome functions. Genes Dev. 2015;29:337-49.

72. Köhler A, Hurt E. Gene regulation by nucleoporins and links to cancer. Mol Cell. 2010;38:6-15.

73. Ikegami K, Lieb JD. Nucleoporins and transcription: new connections, new questions. PLoS Genet. 2010;6:e1000861.

74. Arib G, Akhtar A. Multiple facets of nuclear periphery in gene expression control. Curr Opin Cell Biol. 2011;23:346-53.

75. Schmid M, Arib G, Laemmli C, Nishikawa J, Durussel T, Laemmli UK. Nup-Pl: the nucleopore-promoter interaction of genes in yeast. Mol Cell. 2006;21:379-91.

76. Iwamoto M, Asakawa H, Hiraoka Y, Haraguchi T. Nucleoporin Nup98: a gatekeeper in the eukaryotic kingdoms. Genes Cells. 2010;15:661-9.

77. Pascual-Garcia P, Jeong J, Capelson M. Nucleoporin Nup98 associates with Trx/MLL and NSL histone-modifying complexes and regulates Hox gene expression. Cell Rep. 2014;9:433-42.

78. Oka M, Mura S, Yamada K, Sangel P, Hirata S, Maehara K, Kawakami K, Tachibana T, Ohkawa Y, Kimura H, Yoneda Y. Chromatin-prebound Crm1 recruits Nup98-HoxA9 fusion to induce aberrant expression of Hox cluster genes. elife. 2016;5:e09540.

79. Kehat I, Accornero F, Aronow BJ, Molkentin JD. Modulation of chromatin position and gene expression by HDAC4 interaction with nucleoporins. J Cell Biol. 2011;193:21-9.

80. Rohner S, Kalck V, Wang X, Ikegami K, Lieb JD, Gasser SM, Meister P. Promoter- and RNA polymerase II-dependent hsp-16 gene association with nuclear pores in Caenorhabditis elegans. J Cell Biol. 2013;200:589-604.

81. Chow KH, Elgort S, Dasso M, Ullman KS. Two distinct sites in Nup153 mediate interaction with the SUMO proteases SENP1 and SENP2. Nucleus. 2012;3:349-58.

82. Mendjan S, Taipale M, Kind J, Holz H, Gebhardt P, Schelder M, VermeuIen M, Buscaino A, Duncan K, Mueller J, Wilm M, Stunnenberg HG, Saumweber H, Akhtar A. Nuclear pore components are involved in the transcriptional regulation of dosage compensation in Drosophila. Mol Cell. 2006;21:811-23.

83. Brickner JH, Walter P. Gene recruitment of the activated INO1 locus to the nuclear membrane. PLoS Biol. 2004;2:e342.

84. Rodríguez-Navarro S, Fischer T, Luo MJ, Antúnez O, Brettschneider S, Lechner J, Pérez-Ortín JE, Reed R, Hurt E. Sus1, a functional component of the SAGA histone acetylase complex and the nuclear pore-associated mRNA export machinery. Cell. 2004;116:75-86.

85. Casolari JM, Brown CR, Komili S, West J, Hieronymus H, Silver PA. Genome-wide localization of the nuclear transport machinery couples transcriptional status and nuclear organization. Cell. 2004:117:427-39.

86. Dieppois G, Stutz F. Connecting the transcription site to the nuclear pore: a multi-tether process that regulates gene expression. J Cell Sci. 2010;123(Pt 12):1989-99.

87. García-Oliver E, García-Molinero V, Rodríguez-Navarro S. mRNA export and gene expression: the SAGA-TREX-2 connection. Biochim Biophys Acta. 2012;1819:555-65.
88. Casolari JM, Brown CR, Drubin DA, Rando OJ, Silver PA. Developmentally induced changes in transcriptional program alter spatial organization across chromosomes. Genes Dev. 2005;19:1188-98.

89. Yoshida T, Shimada K, Oma Y, Kalck V, Akimura K, Taddei A, Iwahashi H, Kugou K, Ohta K, Gasser SM, Harata M. Actin-related protein Arp6 influences H2A.Z-dependent and -independent gene expression and links ribosomal protein genes to nuclear pores. PLoS Genet. 2010;6:e1000910.

90. Sarma NJ, Buford TD, Haley T, Barbara-Haley K, Santangelo GM, Willis KA. The nuclear pore complex mediates binding of the Mig1 repressor to target promoters. PLoS ONE. 2011;6:e27117.

91. Stark C, Breitkreutz BJ, Reguly T, Boucher L, Breitkreutz A, Tyers M. BioGRID: a general repository for interaction datasets. Nucleic Acids Res. 2006;34(Database issue):D535-9.

92. Izon DJ, Rozenfeld S, Fong ST, Kömüves L, Largman C, Lawrence HJ. Loss of function of the homeobox gene Hoxa-9 perturbs early T-cell development and induces apoptosis in primitive thymocytes. Blood. 1998;92:383-93.

93. Aubin J, Lemieux M, Tremblay M, Bérard J, Jeannotte L. Early postnatal lethality in Hoxa-5 mutant mice is attributable to respiratory tract defects. Dev Biol. 1997;192:432-45.

94. Seifert A, Werheid DF, Knapp SM, Tobiasch E. Role of Hox genes in stem cell differentiation. World J Stem Cells. 2015;7:583-95.

95. Noordermeer D, Leleu M, Splinter E, Rougemont J, De Laat W, Duboule D. The dynamic architecture of Hox gene clusters. Science. 2011:334:222-5.

96. Rio DC, Ares M, Hannon GJ, Nilsen TW. Purification of RNA using TRIzol (TRI reagent). Cold Spring Harbor Protocol, 2010. 2010:pdb.prot5439.

97. Livak KJ, Schmittgen TD. Analysis of relative gene expression data using real-time quantitative PCR and the 2(-Delta Delta $C(T)$ ) method. Methods. 2001;25:402-8.

98. Carey MF, Peterson CL, Smale ST. Chromatin immunoprecipitation (ChIP). Cold Spring Harbor Protocol, 2009, 2009:pdb.prot5279.

99. Haring M, Offermann S, Danker T, Horst I, Peterhansel C, Stam M. Chromatin immunoprecipitation: optimization, quantitative analysis and data normalization. Plant Methods. 2007;3:11.

100. Landt SG, Marinov GK, Kundaje A, Kheradpour P, Pauli F, Batzoglou S, Bernstein BE, Bickel P, Brown JB, Cayting P, Chen Y, DeSalvo G, Epstein C, Fisher-Aylor KI, Euskirchen G, Gerstein M, Gertz J, Hartemink AJ, Hoffman MM, lyer VR, Jung YL, Karmakar S, Kellis M, Kharchenko PV, Li Q, Liu T, Liu XS, Ma L, Milosavljevic A, Myers RM, et al. ChIP-seq guidelines and practices of the ENCODE and modENCODE consortia. Genome Res. 2012;22:1813-31.

101. Ranade D, Koul S, Thompson J, Prasad KB, Sengupta K. Chromosomal aneuploidies induced upon Lamin B2 depletion are mislocalized in the interphase nucleus. Chromosoma. 2016.

102. Carpenter AE, Jones TR, Lamprecht MR, Clarke C, Kang IH, Friman O, Guertin DA, Chang JH, Lindquist RA, Moffat J, Golland P, Sabatini DM. Cell Profiler: image analysis software for identifying and quantifying cell phenotypes. Genome Biol. 2006;7:R100.

103. Franks TM, Hetzer MW. The role of Nup98 in transcription regulation in healthy and diseased cells. Trends Cell Biol. 2013;23:112-7.

\section{Submit your next manuscript to BioMed Central and we will help you at every step:}

- We accept pre-submission inquiries

- Our selector tool helps you to find the most relevant journal

- We provide round the clock customer support

- Convenient online submission

- Thorough peer review

- Inclusion in PubMed and all major indexing services

- Maximum visibility for your research

Submit your manuscript at www.biomedcentral.com/submit 